\title{
The Influence of Building Geometry on Street Canyon Air Flow: Validation of Large Eddy Simulations against Wind Tunnel Experiments
}

\author{
Maider Llaguno-Munitxa ${ }^{\mathrm{a}, \mathrm{b}, *}$, Elie Bou-Zeid ${ }^{\mathrm{a}}$, Marcus Hultmark \\ ${ }^{a}$ Civil and Environmental Engineering, Princeton University, 41 Olden St, Princeton, NJ 08544, USA \\ ${ }^{\mathrm{b}}$ ETH Institute of Technology in Architecture, Stefano-Franscini-Platz 1, Zurich 8093, Switzerland \\ ${ }^{c}$ Mechanical and Aerospace Engineering, Princeton University, 41 Olden St, Princeton, NJ 08544, USA \\ *Corresponding author at: Environmental Fluid Mechanics Lab, Civil and Environmental Engineering, \\ Princeton University, 41 Olden St, Princeton, NJ 08544, USA, E-mail address: mllaguno@ princeton.edu \\ (M. Llaguno-Munitxa)
}

\begin{abstract}
This study investigates the effect of roof and façade geometry on the mean wind flow and turbulence in street canyons, as well as the ability of numerical simulation techniques in capturing the flow features. Numerical experiments, using the large eddy simulation FLUENT code, have been conducted under neutral stability conditions to test 5 building geometries: i) flat roof, ii) pitched roof, iii) round roof, iv) terraced building and v) building with balconies. Wind tunnel experiments were also conducted for the first three geometries. The simulation and experimental setups were closely matched and both featured configurations consisting of seven building arrays. The results from the physical and numerical experiments concur that (i) in-canyon vortex dynamics and over-canopy flow conditions, are strongly dependent on the geometric features of the buildings, and (ii) pitched and round roof geometries increase in-canyon mean and turbulent velocities, as well as the depth of the shear layer. The findings provide novel insight on the sensitivity of the flow and turbulence fields, as well as the simulation quality, to urban topography, inflow conditions, and the Reynolds number. They also underline the influence on the flow of small-scale features such as balconies, which are often ignored in prior literature.
\end{abstract}

Keywords Building Geometry $\cdot$ Large Eddy Simulations $\cdot$ Street Canyon Design $\cdot$ Urban Ventilation • Wind Tunnel experiments 


\section{Introduction}

As of 2015, more than half of the world's population is living in towns or cities. The number of urban dwellers rose from 729 million in 1950 to 3.9 billion in 2014. By 2050, two out of three humans are expected to be living in urbanized areas (WHO, 2014). The global tendency towards urbanization deteriorates the urban air quality and increases the Urban Heat Island (UHI) intensity, thus amplifying the risk of exposure of citizens to discomfort and health hazards and leading to higher energy demands for cooling (Fernando, 2010; Li and Bou-Zeid, 2013; Li et al, 2014; Oke, 1973).

Air flow plays an important role in determining the urban microclimate and pollutant dispersion (Britter and Hanna, 2003). Regular arrays of cubic buildings have been widely investigated to understand the effect of morphological parameters, such as the plan area density $\lambda_{T}$ and the frontal area density $\lambda_{F}$ on the street canyon air flow dynamics (Grimmond et al, 1998). These parameters have been utilized to describe urban areas as well as to define many empirical urban boundary layer formulas. Using the alternative but related parameters, Oke (1988) distinguished 3 different urban flow regimes, depending on the street width $(S)$ to building height $(H)$ ratios: i) the isolated roughness flow for low urban roughness levels generated by large spacing between buildings $(S / H \gtrsim 3)$ such that the wake of one building does not influence the next building downwind, ii) the wake interference flow where building wakes start to influence the subsequent downwind structures $(1 / 5 \lesssim S / H \lesssim 3)$, and the iii) skimming flow for dense streets where the flow is deviated above the buildings with little penetration into the canyon $(S / H \lesssim 1.5)$. This last regime is very common in urban environments and is typically associated with adverse air quality since the streets are poorly ventilated. Hosker (1987) noted that when $S / H$ is between 1.4 and 2.4 , the wake of one building interferes with the next one downstream, and that when the separation is smaller than 1.4, different vortices are generated within the canyon which depend on the aspect ratio.

The principal steady flow features in a canyon oriented perpendicular to the approach flow are now well known from various published studies (e.g. (Hunter et al, 1990; Kastner-Klein et al, 2004; Kovar-Panskus et al, 2002)). Variations on the different flow regimes have also been reported for example by (Counehan, 1971; Raupach et al, 1980). Counter rotating flow recirculations were observed for narrower street canyons by (Liu et 
al, 2004; Sini et al, 1996). Although to a lesser extent, the influence of the building length (in the stream wise direction, $W$ ) to height ratio, as well as the influence of the angle of attack of the wind flow relative to the main street axis, have also been researched. The angle of attack for example was found to influence the formation of a mean recirculating flow in the street canyon (Yamartino and Wiegand, 1986). Modelling of street canyon pollution was the major topic of the European Research network named "Optimization of Modelling Methods for Traffic Pollution in Streets", with the short acronym TRAPOS (Berkowicz, 2001). Within this network, the influence of traffic movement on turbulence and dispersion of pollutants, the thermal effects on flow modification within street canyons, the sensitivity of the flow and turbulence characteristics to the architecture of the street, the implementation of pollution models for Environmental Impact Assessment studies, and dispersion and transformation processes of traffic-emitted particles were researched.

Most of these previous numerical and wind tunnel studies, developed to model street canyon flow and pollutant dispersion, have been devoted to the exploration of idealized building configurations, i.e. buildings of rectangular shapes and flat roofs. The influence of more complex building geometries, such as variable building heights within the street canyon or variable roof geometries, have more recently been researched but only to a limited extend. Assimakopoulos et al (2003) studied the microscale model MIMO to take into account pollution dispersion in the vicinity of buildings; different aspect ratios were studied by changing the building height, and both street geometry and building height were reported to influence the interaction between air flow inside and above the street canyons. Baik et al (1999) developed similar building height variation studies in a water channel as well as with numerical simulations using Reynolds-averaged Navier Stokes (RANS) simulations. They reported that, for the step-up notch geometry, a vortex is observed in the canyon, while for the step-down notch, two counter rotating vortices were observed. Rafailidis (1997), Rafailidis and Schatzmann (1996) initiated the investigation of the influence of pitched roof building arrays on street flow and pollutant dispersion to conclude that altering the roof geometry can have a bigger impact on urban air quality than modifying canyon aspect ratios. Theodoridis and Moussiopoulos (2000) compared flat and pitched roofs and reported that more complex vortex systems were developed for 
the pitched roof geometries and that when a pitched roof is added to a $S / H=1$ building configuration, a secondary strong vortex is developed at the roof level. Xie et al (2005) studied pitched roofs in combination with flat roofs in different configurations to conclude that in-canyon vortex dynamics and the characteristics of pollutant dispersion are strongly dependent on roof shapes and ambient building structures. Kastner-Klein and Plate (1999) showed results that give evidence that roof shape can be an important factor in determining the vorticity dynamics in the canyon and the intensity of pollutant transport towards the downwind side of the canyon. Kastner-Klein et al (2004) performed wind tunnel studies of wedge-shaped, flat, and pitched roofs, and tested them in various combinations to highlight that roof geometry appears to have a strong influence on the vortex dynamics and therefore also on the canyon ventilation mechanism. Huang et al ( 2009) revealed that inclined roofs affect in-canyon vortex dynamics and the pollutant distribution. Yassin (2011) found that roof slopes are important for street canyon air flow and pollutant concentration. Kellnerova et al (2012) developed wind tunnel measurements using the PIV technique to compare the flow features generated by the presence of flat and pitched roofs. They also reported that given the presence of the sharp crease of the pitched roof, behind the roof top, an intense vortex is generated. Takano and Moonen (2013) studied the critical roof angles that generated a mean recirculating flow in the street canyon. The number of vortices generated within the street canyon was found to be dependent on the roof angle.

As described above, slanted and pitched roofs have been quite extensively studied in prior literature; however, this is not the case for round roof geometries. Dezső-Weidinger et al (2003) performed wind tunnel studies of an array of linear buildings with a round roof geometry. However, given that the main focus of that study was the exploration of a new PIV/PTV measuring technique, the flow physics obtained from the study were not extensively reported. More recently, Huang et al (2014) studied the influence of 5 different building roof geometries, the vaulted roof, the trapezoidal roof, the slanted roof, the upward wedged roof and the downward wedged roofs, as well as three different roofheight to building-height ratios for the upstream building roof. They reported that the shape and height of an upstream roof have significant influence on flow pattern and pollutant distribution in an urban canyon. However, their studies used the RANS 
approach with a $k-\varepsilon$ model, which hindered detailed analyses and validation of higherorder turbulence statistics.

Similarly, the influence of smaller roughness scale geometrical features, such as the presence of terraces in building cornices or balconies, on street canyon flow statistics have not yet been studied in detail. Therefore, the understanding as to which extent small roughness scale elements influence the street canyon air flow characteristics is yet to be fully developed. Salizzoni et al (2008) conducted wind tunnel experiments to investigate this question over a series of street canyons and for various street canyon aspect ratios; they reported that the tested small-scale roughness elements (2D roughness elements that were added to the top of the building arrays) only influence the dynamics in the skimming flow regime, and only if they are sufficiently large to interact with the eddies generated by the building that have a scale $\sim H$. However, further research on different geometrical characteristics is necessary to understand the influence of smaller roughness scale elements such as façade or urban elements on the in-canyon and above-canyon flow conditions. Zajic et al (2011), based on data acquired from an atmospheric measurement campaign developed in Oklahoma City during summer 2003 and which therefore would include such small features, reported on the dependence of turbulence quantities on incoming wind direction and time of the day. However, as they noted, the complexity of the flow configurations causes the flows to be largely site dependent. This limits the ability to understand the fundamental features of the flow, which as they state "is best obtained using laboratory and numerical investigations based on idealized geometries" (Zajic et al, 2011). Savory et al (2013) also pointed out the limitations when operating on overly detailed or "realistic" setups as they called it, given that the results become too site specific. On the other hand, an over simplification can also prove limiting. The balance we strike in this paper is to analyse the flow in the last canyon of a six-identical-canyons configuration. Many urban neighbourhoods have very homogeneous building heights and roof shapes, and the flow over such areas can be seen to be "in equilibrium" with the underlying surface (i.e. flow profiles are self-similar). This implies that the inflow to each canyon (except the few ones on the edges) is similar and is determined by the homogeneous topographic features of the neighbourhood. 
This choice is consequential since the influence of the upstream building geometries, as well as the inflow conditions, have been shown to be quite important when studying the mean and turbulent statistics within the urban street canyon. Brown et al (2000) developed wind tunnel studies of a street canyon configuration composed of a sevenbuilding array, and described the relevance of the upstream building configuration given the variability of the flow features from canyon \#1 to canyon \#6. They reported that only after canyon \#3 or \#4 can the flow be considered in equilibrium. Salizzoni et al (2011) also studied the effect of upstream array building configurations by varying the width of the canyons upstream while maintaining the square geometry of the test canyon. They reported that momentum transfer is due to coupling between the instabilities generated in the shear layer above the canyons and turbulent structures in the ABL. Therefore, if the building arrays are widely spaced, it is likely that the levels of intensity and scales of turbulence in the ABL will be reduced. Savory et al (2013) described the importance of wind tunnel modelling of the flow, with the aim to achieve approach flow conditions that most closely represent the full-scale to study the mean, turbulence and concentration characteristics. Furthermore, in their studies, Savory et al (2013) also focused on the upstream roughness elements and studied two cases based on isolated roughness segments (3D roughness elements), and building array type (2D roughness elements) and reported that the details of the approach flow conditions have a strong effect on the canyon flow. They recommend utilizing 3D upstream roughness arrangements when aiming to match "realistic" urban-like environments given that 2-D building arrays tend to generate a 2D flow behaviour. Similarly, Blackman et al (2014) compared the effect of upstream flow using building cubes or building arrays and reported that the mean streamwise velocity is higher for $3 \mathrm{D}$ than $2 \mathrm{D}$ configurations, the spatially averaged shear however was reported to be lower for the 3D case. The Turbulent Kinetic Energy (TKE) profiles were also reported to be different. This previous research underlines the difficulty in defining a "realistic" inflow since changes in the upstream geometry invariably alter the downstream flow and since in real cities the upstream conditions are highly variable. This motivates our use of an "equilibrium" inflow, which is the most probable in the real-world. This approach is a departure from previous studies where all 
upstream models were usually represented as flat roof configurations, and only the roofs in the canyon under study were modified.

To summarize, previous research has indicated that urban flows are highly sensitive to the geometry of the underlying buildings and the upwind flow conditions, but the extent to which small-scale building features need to be included remains uncertain. Therefore, advancing our understanding of urban flows requires the ability to study a large range of idealized and realistic setups. This can be most easily achieved using numerical simulations, but the codes need to be validated for various canonical building shapes since success in simulating flat roofs does not necessarily extend to other more complex topographies (as we will show in this paper). To help address these open challenges, the overall aims of the present paper are:

(1) To evaluate the Fluent LES code for various street canyon building geometries through idealized wind tunnel experiments, and assess its capability to capture the influence of geometrical attributes.

(2) To understand the influence of roof and smaller-scale building geometrical features, such as balconies, on the in-canyon and over-canopy flows, and assess the need to include them in fluid dynamics studies of urban terrain.

(3) To understand flow equilibration as it transitions from the upstream inflow conditions to a homogeneous urban canopy, and therefore evaluate whether the obtained statistics are representative of large urban neighbourhoods with homogeneous building heights and roof shapes.

\section{Investigation Setups and Methods}

The wind-tunnel and numerical studies presented in this paper focus on an array of seven two-dimensional buildings as the basic configuration. The buildings are perpendicular to the mean wind direction, and thus the resulting six street canyons are aligned in the crossstream direction. This configuration was used as a reference case for a systematic study of building geometry variations. The building geometries that have been studied are: i) the flat roof ii) the pitched roof, iii) the round roof, iv) the terraced building and v) building with façade balconies. The model dimensions have been kept proportional for both the numerical simulations and wind tunnel experiments to ensure geometric 
similarity by maintaining the same ratios between all the linear dimensions, including the spacing and height of the surface geometries.

Three inflow conditions have been tested for the numerical cases. Initially, the numerical simulations have been performed by imposing a laminar and uniform inflow condition (inlet \#1), designed to match the inlet boundary conditions of the wind tunnel for validation purposes; laminar uniform inflow is the easiest to characterize and replicate in simulations. Once the validation process has been performed, the same geometrical configurations have been numerically tested with a new inlet condition, where a log profile and turbulent conditions have been imposed (inlet \#2), aiming to achieve the flow conditions that are more representative of the approach flow present in a generalized urban-like scenario. Finally, Brown et al (2000) inflow conditions have been replicated (inlet \#3), to validate our LES data against their wind tunnel measurements. Inlet \#3 has only been applied to the flat roof geometry (this is the only geometry that (Brown et al, 2000) studied, and inlet \#1 and \#2 have been applied to all tested geometries.

Figure 1 depicts the LES results (detailed later) comparing the three inflows for all street canyons. The central vertical axes (the midpoint in the cross-stream direction) mean velocity and turbulence statistics for the round roof geometries are plotted. Figure 1a shows the mean velocity $(U)$ profiles for all three inlet conditions, in all 6 street canyons as well as at $2 \mathrm{H}$ and $4 \mathrm{H}$ upstream of the first model and downstream of the last model. Figure $1 \mathrm{~b}$ shows the LES results for the root mean square velocity $=\sqrt{{ }_{u}^{2}+{ }_{v}^{2}+{ }_{w}^{2}}$. As reported by Brown et al (2000), self-similarity of the mean and turbulent profiles is observed for all cases after canyon \#4. This confirms that the results from the last canyon are representative of an infinite array and very weakly dependent on upstream inflow conditions; therefore, all measurements, validations, and analyses focus on the last downstream canyon (referred to as canyon \#6). 

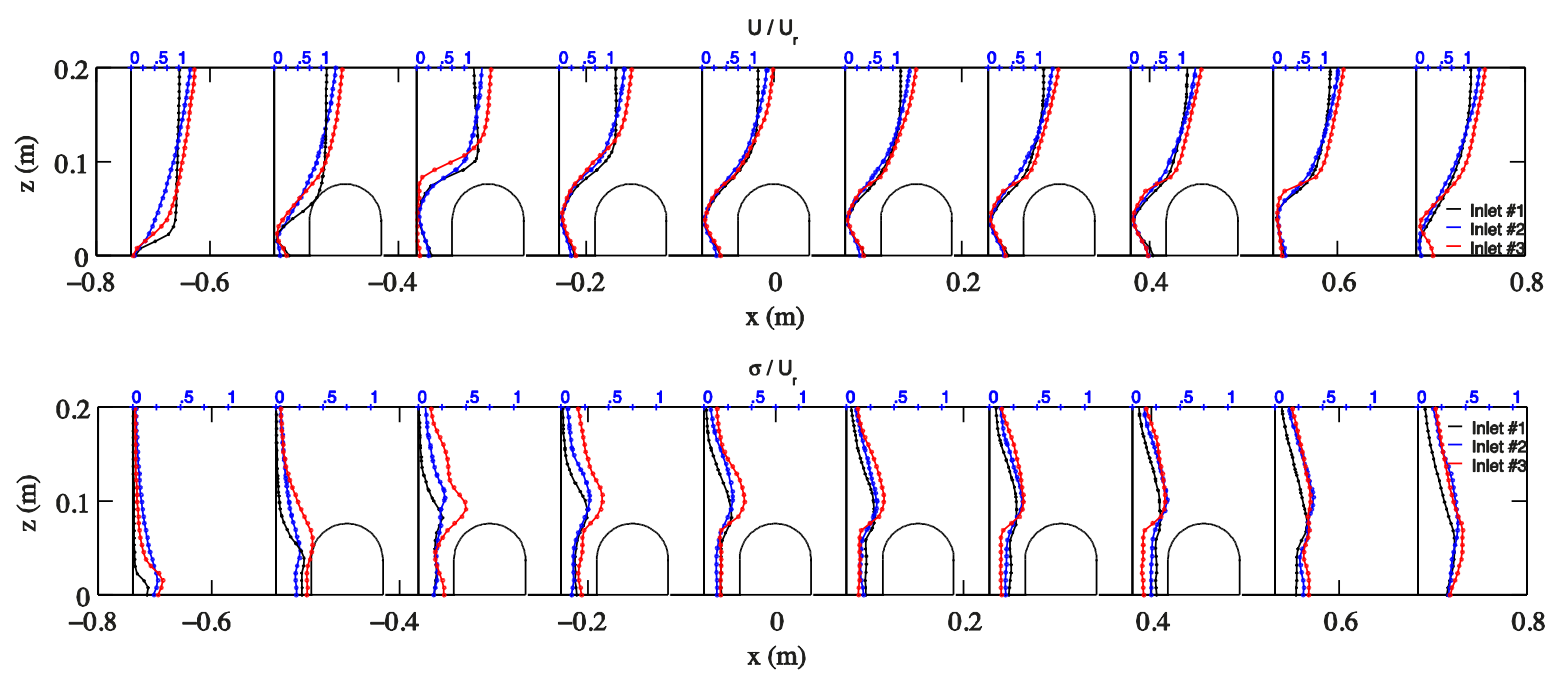

Fig 1 Top figure: mean velocity magnitude $U$ for the round roof geometry (from LES) in m s $\mathrm{s}^{-1}$ for the 6 canyons, depicting the fully developed regime established in the last street canyon (flow is from left to right). The results from all three inlet conditions are compared. The characteristics of the inlet conditions are detailed in Sect. 2.2.2. The bottom figure shows the results for $(\sigma)$ also for all inlets and for the round roof geometry. The results are normalized by the $U_{r}$, the upstream flow velocity at $2.5 \mathrm{H}$ above the bottom wall.

\subsection{Experimental setup}

For the wind tunnel experiments, high density expanded polyurethane foam has been used to build the models $(0.07 \mathrm{~m} \times 0.07 \mathrm{~m}$ for the flat roof). The overall height of the pitched and round roofs was also $0.07 \mathrm{~m}$. The models were sanded to guarantee a hydrodynamically smooth surface, and they were extended side to side covering all the width of the test section to obtain a quasi-2-D flow inside the street canyons (see Fig. 2). The closed loop wind tunnel located in Princeton University's Instructional Fluid Dynamics Laboratory was used. The tunnel is a modified version of a wind tunnel model 407-A manufactured by Engineering Laboratory Design, Inc. The test section of the tunnel is $0.61 \mathrm{~m}$ (height) $\times 0.91 \mathrm{~m}($ width $) \times 2.64 \mathrm{~m}$ (length). The flow passes through a conditioning area containing a honeycomb, a screen, and a rectangular 4.2:1 contraction area. The tunnel includes an axial flow fan with variable pitch blades that enables a velocity range of 3.0 to $55 \mathrm{~m} \mathrm{~s}^{-1}$. Due to the high forces exerted on the models at high wind speeds, the tests were performed at up to $7.5 \mathrm{~m} \mathrm{~s}^{-1}$. The tunnel is equipped with a heat exchanger located upstream of the flow conditioning sections for flow temperature control (Jimenez, 2007). 
The walls of the tunnel are built out of plexiglass surfaces that enable full visual access to the whole test section. The test section walls are removable for full accessibility for the installation of the physical models. The tunnel also provides an accessible slot of $0.05 \mathrm{~m}$ width $\times 1 \mathrm{~m}$ length, in the centre of the test section along the wind tunnel axis, through which we introduced the measuring probes. The slot has been sealed for the duration of the experiments, except for the location where the probe is introduced, such that only minimal leakage occurs.

Measurements were conducted at two free-stream velocities $U_{r}$ of $3.5 \mathrm{~m} \mathrm{~s}^{-1}$ and $7.5 \mathrm{~m}$ $\mathrm{s}^{-1}$, corresponding to Reynolds numbers, based on the length scale of the buildings $L_{r}=$ $0.07 \mathrm{~m}$ and an air viscosity $v=1.5 \times 10^{-5} \mathrm{~m}^{2} \mathrm{~s}^{-1}$, of $R e_{M L}=1.77 \times 10^{4}$ and $3.8 \times 10^{4}$, respectively. The corresponding Kolmogorov length scales ranged from $4.94 \times 10^{-5} \mathrm{~m}$ to $2.79 \times 10^{-5} \mathrm{~m}$, and the characteristic large eddy turnover time ranged between 0.0110 and $0.0217 \mathrm{~s}$ and (see Table 1). Upstream of the street canyon models, the domain is $5 H$ long between the inflow and the first building boundary, and $15 H$ long downstream from the last building to the outlet. For comparison with real-world buildings, we consider a length scale and reference velocity for prototypical buildings and atmospheric conditions as $L_{p}=$ $15 \mathrm{~m}$ and $U_{p}=2 \mathrm{~m} \mathrm{~s}^{-1}$ with $R e_{p}=2.0 \times 10^{6}\left(v\right.$ of $\left.1.5 \times 10^{-5} \mathrm{~m}^{2} \mathrm{~s}^{-1}\right)$. Therefore, the length scale ratio $L_{r} / L_{p}$ between the model and the real building is $1 / 200$; we will come back to the effect of the Reynolds number $(R e)$ disparity this leads to later in the paper.

The model scale has been made as small as possible to minimize the pressure gradients induced by the blockage effect. However, given the necessity to access the street canyon with the hot wire probe, the minimum size of the street canyon width $S$ was constrained to remain larger than $0.07 \mathrm{~m}$; the resulting blockage ratio was $11 \%$. In order to evaluate the blockage effect the relatively large scale of the models could induce, two LES studies were developed with two different domain heights of 0.61 and $0.77 \mathrm{~m}$ tall respectively. The results obtained from the two LES studies have shown that the velocity differences induced by the blockage effect are not negligible upstream (first canyon); however, further downstream (in canyon \#6) where the research is primarily focused, the blockage effects became insignificant. Therefore, the domain size of the wind tunnel can be considered acceptable for the intents and purposes of this study where we are interested in homogeneous urban roughness. Moreover, the same domain size is 
reproduced in the LES studies described in the following section such that the blockage effects do not influence the validation. Three physical models were tested in the tunnel with different roof geometries as depicted in Figure 2 (recall that in the numerical simulations we consider 5 geometries).

Table 1. Experimental setup parameters

\begin{tabular}{|l|c|c|}
\hline & $U_{r}=3.5 \mathrm{~m} \mathrm{~s}^{-1}$ & $U_{r}=7.5 \mathrm{~m} \mathrm{~s}^{-1}$ \\
\hline Turbulent kinetic energy dissipation $=U_{r}^{3} / H$ & $564 \mathrm{~m}^{2} \mathrm{~s}^{-3}$ & $5551 \mathrm{~m}^{2} \mathrm{~s}^{-3}$ \\
\hline Kolmogorov length scale $=\left({ }^{3} /\right)^{1 / 4}$ & $4.94 \times 10^{-5} \mathrm{~m}$ & $2.79 \times 10^{-5} \mathrm{~m}$ \\
\hline Kolmogorov time scale ${ }_{n}=(/)^{1 / 2}$ & $1.63 \times 10^{-4} \mathrm{~s}$ & $5.19 \times 10^{-5} \mathrm{~s}$ \\
\hline Kolmogorov time/sampling rate ${ }_{n} / s_{r}$ & 9.6 & 3.1 \\
\hline Characteristic eddy turn-over time $t_{0}=l_{r} / u_{r}$ & $2.17 \times 10^{-2} \mathrm{~s}$ & $1.10 \times 10^{-2} \mathrm{~s}$ \\
\hline
\end{tabular}
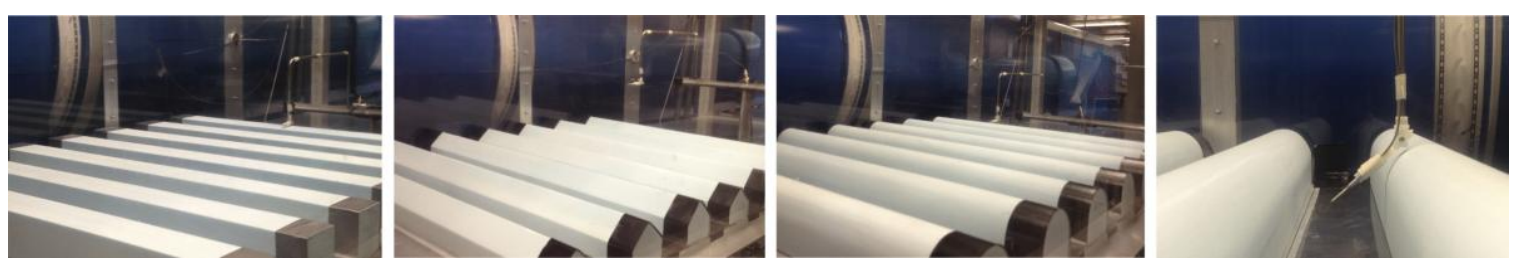

Fig 2 Wind tunnel setup: a) flat roof b) pitched roof c) round roof and d) close up view in canyon \#6 


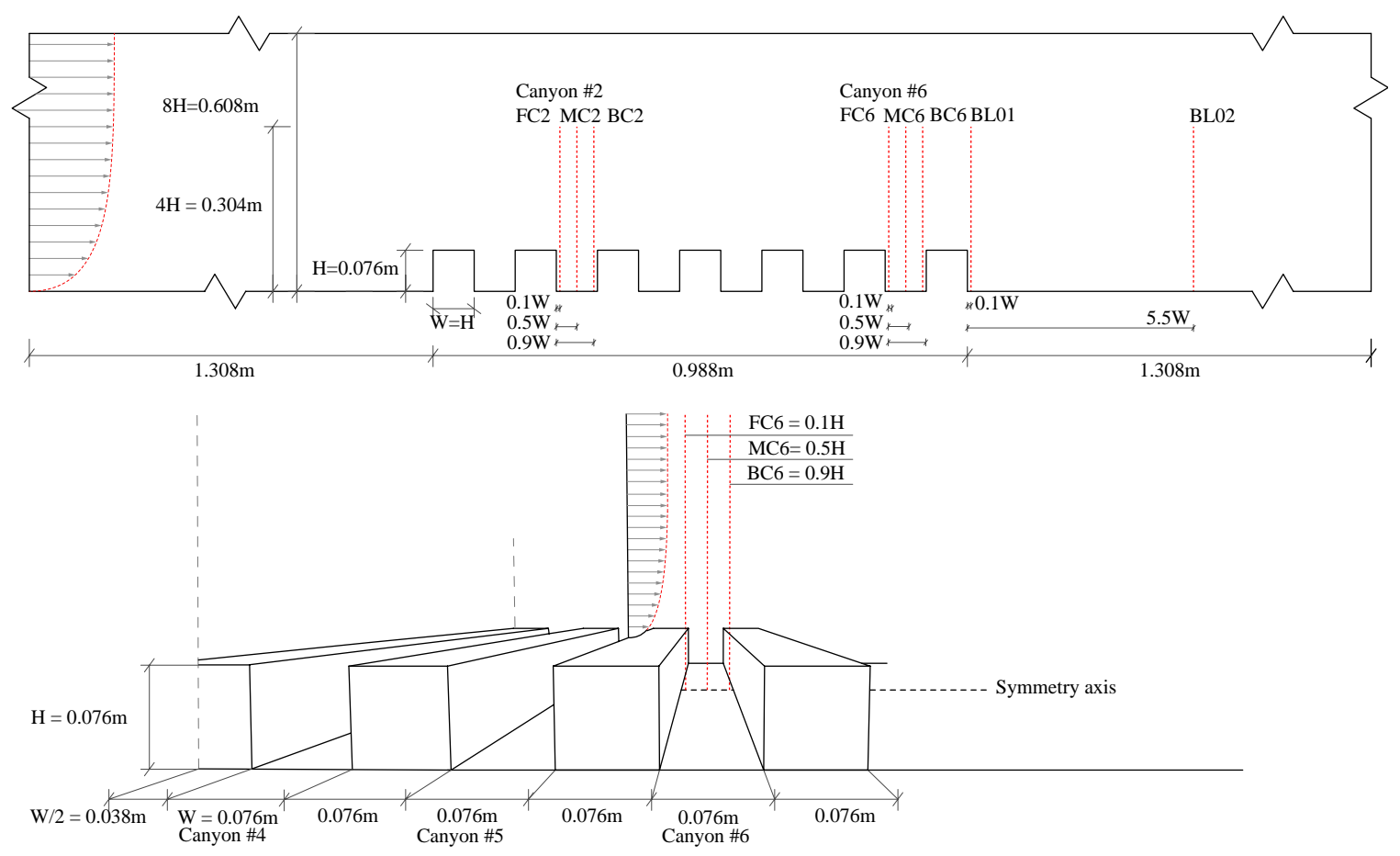

Fig 3 Wind tunnel and LES domain setup and vertical profile measuring locations. In the wind tunnel, flow measurements have been collected for vertical rakes FC6, MC6 and BC6. In LES, apart from the rakes in canyon \#6, further vertical rakes have been recorded as depicted in the figure. In the comparison to the data of (Brown et al, 2000), results are also evaluated in canyon \#2 as well as in BL01 and BL02 since measurements in that canyon are available in that study.

\subsubsection{Measuring technique}

The flow velocity measurements were conducted using a hot-wire anemometry system operating in constant temperature anemometer (CTA) mode, composed of i) a single hotwire sensor exposed normal to the air in motion, ii) a control unit which maintains the temperature of the hot-wire sensor constant, compensating for the fluctuations induced by the air stream, and iii) the application software for data acquisition and analysis. The hotwire was operated by a Dantec Streamware constant temperature anemometer system. An etched Wollaston wire of $d=5 \mu \mathrm{m}$ in diameter (90\% Pt 10\% Rh) has been used, the sensing length $l$ of which ranged from $1 \mathrm{~mm}$ to $2 \mathrm{~mm}$. Therefore, the criterion for $l / d>$ 200 (Ligrani and Bradshaw, 1987) has been followed in this experiment.

The Hot-wire probe calibrations for the velocity reference were performed before and after the data of each rake were gathered using a Pitot tube with a Setra manufacturing (model 339) electronic manometer. The probe spent 2 min at every point, and each rake had 18 points. A minimum of two calibrations were conducted for each measurement to 
reduce the time between calibrations and measurements. The calibrations were conducted in the free-stream at $4 \mathrm{H}$ above the street canyon models and for 7 different velocities at zero pitch angle. Before the single-wire calibration was conducted, the electronic drift of the Pitot tube pressure transducers was measured and generally found to be insignificant. Calibration data were fitted using King's law assumption. If the difference between the calibration curves before and after each measurement was larger than 5\% in velocity, the measurements were discarded. The tunnel temperature was regulated such that no temperature correction was deemed necessary.

While hot wires prove adequate for turbulence measurements because of their high temporal resolution, their downside is that they only provide measurements at one point at a time. To sample at multiple points in canyon \#6, the hot-wire probe was moved with the aid of three robotic stages that cover the $x, y$ and $z$ axis for a three-dimensional mapping. A grid of 5 sampling points in $x$ (streamwise), 3 sampling points in $y$ (crossstream) and 18 sampling points in $z$ (vertical) was covered for each experiment. The sampling area has been constrained between $0.5 H$ (it was impractical to reach the wind tunnel surface) and $3 \mathrm{H}$ in $\mathrm{z}$ to understand the flow variability below and above the urban canopy layer (UCL). This resulted in 270 points sampled per experiment. At each measurement position, time histories of streamwise velocity, $U(t)$, were acquired at a rate of $60 \mathrm{kHz}$ for $120 \mathrm{~s}$ to guarantee that both the Kolmogorov and integral length scales were captured. Note that since the building-scale eddy turnover time was $\lesssim 2 \times 10^{-2}$ s (see Table 1), over 6000 large eddies were sampled at every point, guaranteeing statistical convergence of the results.

\subsubsection{Fog experiment}

Seeking a qualitative visualization to understand the flow behaviour when interacting with the different building geometries, a mineral oil fog visualization analysis has been performed. The fog source has been positioned in the middle of the street canyon to visualize the air exchange patterns between the canyon and free stream flows. A small diameter tube was used to release the gas, ensuring that flow disturbance by the tube does not generate larger local pressure fluctuations that would influence the fog release (see Fig. 4). The fog generator keeps the pipe full of fog at all times and the fog is released by 
the suction force induced by the incoming air flow which channels the fog out from the tube. While we could not ensure that this setup result in an entrance fog velocity that is insignificant to the transport, we note that the use of these results is only qualitative and the patterns observed do agree with the numerical simulations as will be shown later.

LED light sources were placed in a linear configuration, on the top and bottom of the test section outside of the tunnel and perpendicular to the street canyon. In order to produce a vertical light sheet in the street canyon section flow, the LED light beams have been channelled through 4 parallel vertical foam boards $(0.75 \mathrm{~m}$ (height) $\times 0.75 \mathrm{~m}$ (width) $\times 0.015 \mathrm{~m}$ (thick)), 2 on top of the test section and 2 below. The boards have a separation of $0.015 \mathrm{~m}$ between them to let the LED light source in, and direct the light along the panel length to reduce the angle of the light beam (focus it) when it reaches the street canyon.

To guarantee full blackout, all surfaces of the test section were covered with a black film. A slot at height $1 \mathrm{H}$, aligned with the centre of the street canyon, was left open to locate the camera objective. A video of $60 \mathrm{~s}$ was recorded for each geometry. The instantaneous photos displayed in Figure 4 characterize the dominant flow features, which were consistent for each roof at various times, but differed for various roof types. A video visualization for the 3 roof geometries has also been made available. As can be seen in the figure, the flow patterns are quite different and some coherent features can be observed even in this instantaneous shot. We will elaborate on these differences in Sect. 3.
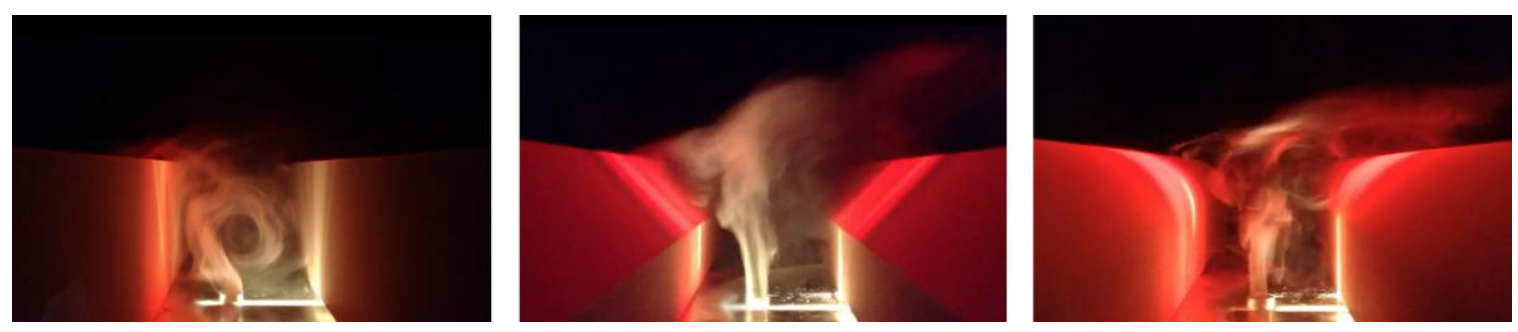

Fig 4 Fog visualizations for the a) flat roof b) pitched roof c) round roof; mean flow is from left to right. (The flow travels from left to right)

\subsection{Numerical setup}


The LES technique strikes a balance between the more computationally demanding direct numerical simulation approach that resolves all turbulent scales, and the less accurate Reynolds-averaged Navier Stokes simulations where only the mean flow is resolved and all turbulence scales are parameterized (Georgiadis et al, 2010; Pope, 2000). LES directly calculates the large-scale turbulent structures, larger than the grid or filter scale, and only requires modelling of the smaller scales. The technique has thus become the tool of choice for turbulent flow simulation in many engineering and environmental applications, including urban flows (Bou-Zeid et al, 2009; Inagaki et al, 2012; Xie and Castro, 2009; Yaghoobian et al, 2014). Therefore, we use LES to numerically simulate the experimental setup, with the aims of validating the model and augmenting the experimental data with the 3D fields of all parameters that the simulations can provide, as well as test the influence of geometry on the capabilities of the simulations. Here we use the LES solver of Fluent 15 (Ansys Inc.).

The filtered LES continuity and momentum equations solved by Fluent LES reduce for incompressible flow to:

$$
\begin{gathered}
\frac{\partial \overline{u_{i}}}{\partial x_{i}}=0 \\
\frac{u_{i}}{t}+\frac{}{x_{j}}\left(\overline{u_{i} u_{j}}\right)=\frac{1}{\frac{p}{x_{i}}}+2 \frac{\bar{S}_{i j}}{x_{j}} \frac{\overline{i j}_{x_{j}}}{}
\end{gathered}
$$

$u_{i}$ is the full velocity vector in index notation, which can be Reynolds-decomposed into a mean $U_{i}$ (capital letters always denote means) and a perturbation $u^{\prime}$ (primes always denote turbulent perturbations). $x_{i}$ is the position vector and $p$ the pressure. The overbar denotes filtering; $S_{i j}$ is the strain rate tensor; and $\tau_{i j}$ is the subgrid scale (SGS) stress tensor. Only the deviatoric part of $\tau_{i j}$ is modelled in Fluent via an SGS eddy viscosity $\left(v_{t}\right)$ closure, $\quad{ }_{i j}^{D}=-2{ }_{t} \bar{S}_{i j}$, while the isotropic part is added to the pressure as is common in many LES codes (e.g. (Bou-Zeid, 2005)). Specifically, the Algebraic Wall-Modelled LES (WMLES) subgrid SGS model has been used for the different numerical simulations. WMLES is a hybrid RANS/LES method, where RANS is used in regions where the turbulence is in equilibrium, such as in wall boundary layers, and LES is used where non- 
equilibrium occurs, typically away from walls. This approach relaxes the need to resolve the viscous sublayer (wall-resolved LES, see Pope 2000) and drastically reduces the computational cost of the simulations. The SGS eddy viscosity $v_{t}$ in WMLES is calculated through the formulation of (Shur et al, 2008), which combines a mixing length model for the RANS region, a modified Smagorinsky model (Smagorinsky, 1963) for the LES region, and a wall damping function (Piomelli et al, 1988). The resulting expression of $v_{t}$ hence features a hybrid mixing length scale (Fluent 15 Theory Guideline(ANSYS, 2013)):

$$
\left.{ }_{t}=\min \left[\left(k d_{w}\right)^{2},\left(C_{\text {smag }}\right)^{2}\right] \bar{S}\left(1 \quad \exp \mid\left(\frac{y^{+}}{25}\right)^{3}\right)\right),
$$

where $d_{w}$ is the distance to the wall; $S$ is the strain rate magnitude; $k=0.41$ is the von Kármán constant; $C_{S m a g}=0.2$ is the Smagorinsky model constant; and $y^{+}$is the distance normal to the wall in viscous units. The LES model uses a modified grid filter scale to account for the grid anisotropies in wall-modelled flows:

$$
=\min \left(\max \left(C_{w} d_{w} ; C_{w} h_{\max } ; h_{w n}\right) ; h_{\max }\right) .
$$

Here, $h_{\max }$ is the maximum edge length of the cell, $h_{w n}$ the wall-normal grid spacing, and $C_{w}=0.15$ a constant.

The pressure-velocity coupling follows the SIMPLEC scheme. The spatial discretization for the momentum and energy equations uses the Least Squares Cell based method for the gradient, standard method for the pressure, and bounded central differencing for the momentum. The time advancement uses an implicit second-order scheme. The dimensional time step size has been set to $d t=0.00025 \mathrm{~s}$ and the cases have been run for 20,000 time-steps. The time-step ensures that the Courant-Friedrichs-Lewy (CFL) number (Courant et al, 1928) is always smaller than $1(\approx 0.32)$ at all grid points. Since the eddy turnover time is $\sim 0.0076 \mathrm{~s}$ due to the small spatial scale of the model (see Table 2), each simulation includes about 600 eddy turnovers. The initial 10,000 timesteps were for warm-up and were not considered in the calculations to remove the influence of the initial conditions. The results shown are hence averaged over the last 10,000 time steps, equivalent to about 300 eddy turnovers (about $2.5 \mathrm{~s}$ in physical time) which is sufficient for the statistics to converge. 
Since Fluent computes the molecular viscous terms in the momentum equations, and captures the influence of viscosity on the flow-wall interaction in the WMLES, the simulations should in principle reproduce the correct $R e$ of the flow given the velocity and spatial scale inputs (it is not an inviscid wall-modelled LES). The simulations should thus also have preserved the dynamic similarity vis-à-vis the experiments since the chosen $R e$ are very close.

Table 2. Numerical setup parameters

\begin{tabular}{|l|l|}
\hline Front/top area density & $\lambda_{f}, \lambda_{p}=0.076 \mathrm{~m}$ \\
\hline Inlet velocity & $U_{r}=10 \mathrm{~m} \mathrm{~s}^{-1}$ \\
\hline Local grid scale & $\Delta=0.0076 \mathrm{~m}$ \\
\hline Flow time over full domain & $T_{F D}=L U_{r}^{-1}=0.37 \mathrm{~s}$ \\
\hline Time computed & $T_{c}=3.75 \mathrm{~s}$ \\
\hline Building-scale eddy turnover time & $T_{T 0} \approx H U_{r}=0.0076 \mathrm{~s}$ \\
\hline
\end{tabular}

\subsubsection{Computational grid}

Grid sensitivity analysis has been performed for two of the studied geometries cases, the flat roof and the round roof geometries. Three grid resolutions, composed by isotropic hexahedral cells, have been tested for each of the cases. Isotropic meshes have been preferred so that grid stretching and compression is negligible in regions of high gradients, to keep the truncation error small (Franke et al, 2007). The number of cells in the different meshes were $8.2 \times 10^{6}, 4.6 \times 10^{6}$ and $2.4 \times 10^{6}$ cells. The results obtained with the two higher resolution meshes are reasonably close for the mean velocity profiles as displayed in Figure 5a. Only the resolved parts of the variances are provided as output by Fluent and used or plotted throughout this paper. Therefore, the root mean-square (rms) velocity $\sigma$ will not converge since as the grid is refined more scales are resolved by the LES, leading to a continuous increase in $\sigma$. However, the results in Figure $5 \mathrm{~b}$ suggest that the trends of the profiles are consistent across all resolutions. Therefore, the intermediate grid resolution has been utilized for the studied building geometries. 


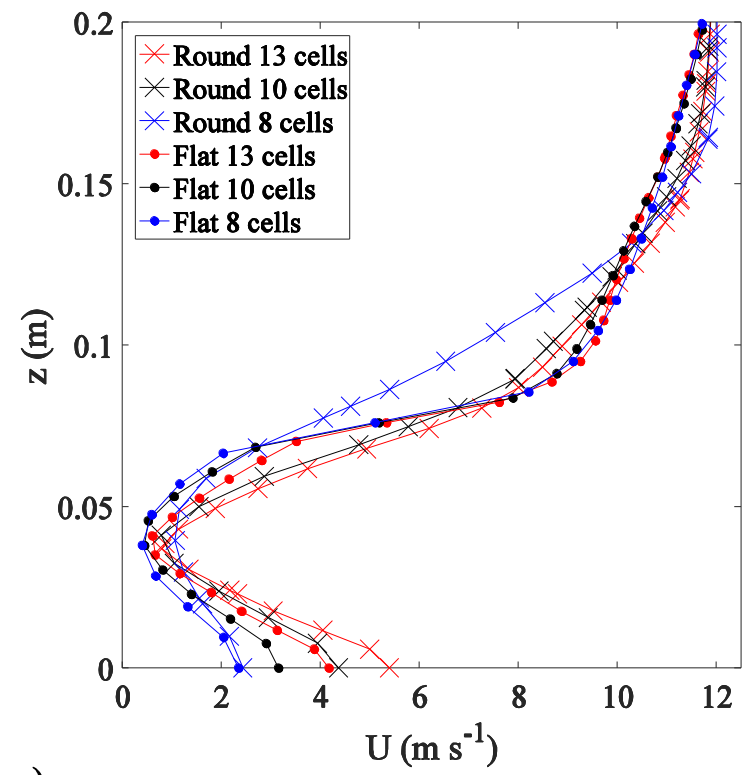

a)

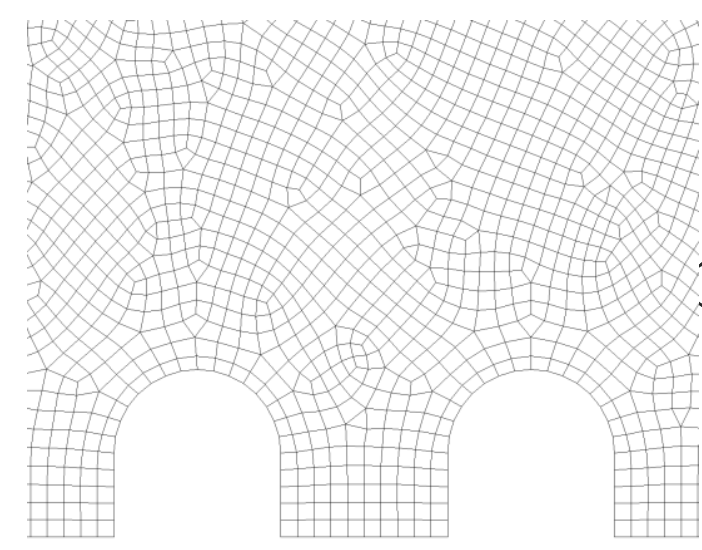

c)

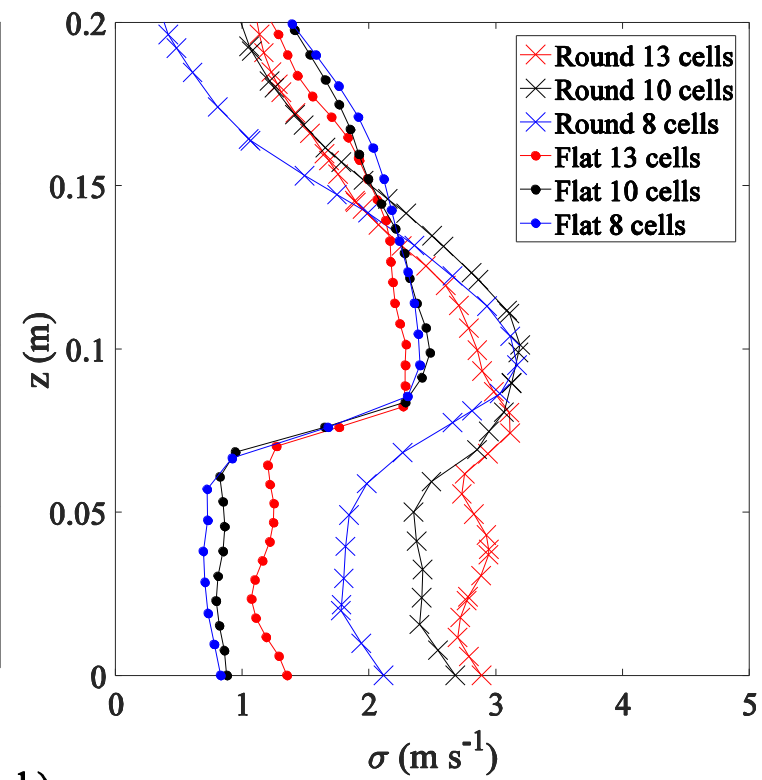

b)

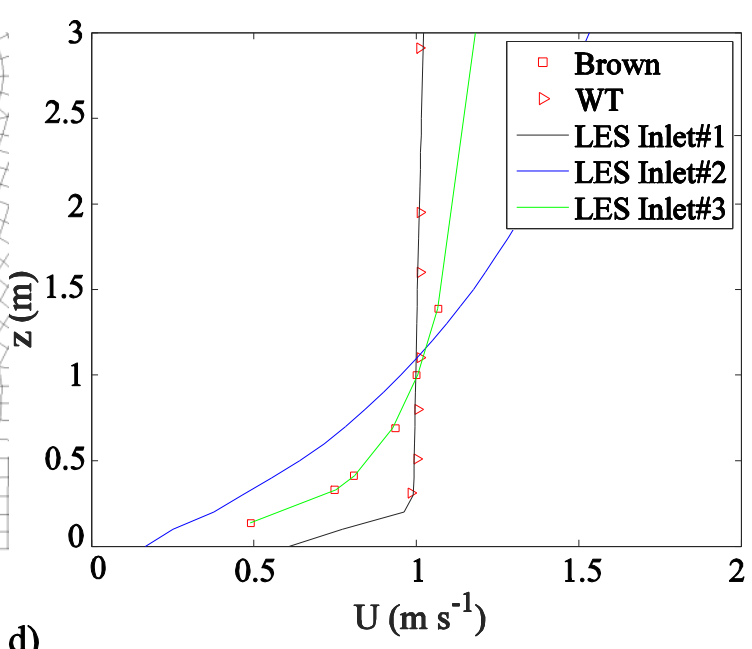

Fig 5 a) The mean velocity profiles for the 3 grid resolutions and 2 geometries. b) The root mean square profiles for the 3 grid resolutions and 2 geometries. c) Round roof geometry mesh resolution example d) The mean velocity profiles for the studied inlet conditions: Brown's inlet profile, the wind tunnel inlet profile, Inlet \#1, Inlet \#2 and Inlet \#3.

This adopted resolution results in grid cells of $0.007 \times 0.007 \times 0.007 \mathrm{~m}$, equivalent to having about 10 grid points spanning each building facet. The flat roof mesh domain is composed of $80 \times 120 \times 490(H \times W \times L)$; that is about $4.704 \times 10^{6}$ grid nodes. The pitched roof mesh domain is composed by $4.8 \times 10^{6}$ grid nodes. The round roof mesh domain is composed by $4.8 \times 10^{6}$ grid nodes. The terraced model is covered by $4.7 \times 10^{6}$ grid nodes, and the one with balconies by $4.7 \times 10^{6}$ grid nodes. 
The computational domain is $3.724 \mathrm{~m}$ long, $0.912 \mathrm{~m}$ wide and $0.608 \mathrm{~m}$ tall, mimicking the cross section of the wind tunnel. Between the first street canyon model and the inlet there are $1.308 \mathrm{~m}$, and the same distance is used from the last canyon model to the outlet (there is no need to match the wind tunnel length). A sample of the instantaneous flow fields generated for two of the studied geometries is depicted in Figure 6. Note that these fields are from simulations with a laminar inflow (Inlet \#1) and thus the incoming turbulence levels are very low. As in the wind tunnel visualizations of section 2.1.2, the results suggest a strong influence of the geometry on the flow patterns and the circulation in the canyon.
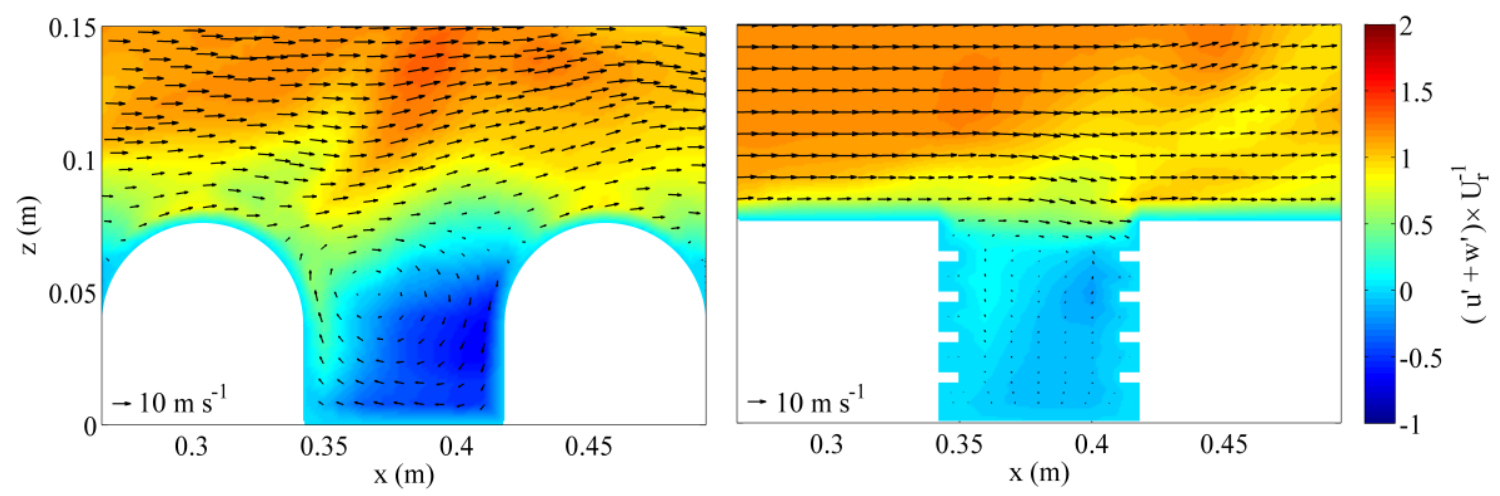

Fig 6 LES instantaneous velocity vector plots overlaid on a pseudo-colour plot of the velocity magnitude: a) Round roof b) Building with balconies. The results are normalized by $U_{r}$, the upstream flow velocity at 2.5H. All plots use inlet conditions \#1.

\subsubsection{Boundary conditions}

Hydraulically smooth walls were assumed for the ground, top and side walls, as well as all building surfaces (consistent with the mixing length formulation given in Eq. 3). Fluent's "outlet" boundary condition has been specified at the downstream end of the domain; it extrapolates the needed gradients from within the domain to the boundary to limit the impact of this boundary on the rest of the flow.

As stated in the introduction, three inlet conditions have been tested for all studied building geometries. Here we provide further details about these inflows:

a) Inlet \#1: Designed to match the idealized wind tunnel inlet conditions for validation purposes. A fully laminar flow with a uniform inlet velocity of $10 \mathrm{~m} \mathrm{~s}^{-1}$ has been defined. This inflow will be the default one used for all results and figures unless otherwise noted. 
b) Inlet \#2: Has been used to reproduce flow conditions that are more likely in a real urban environment. A turbulent inflow with an imposed log profile with high roughness length (mimicking rough urban terrain also upwind) has been implemented aiming to reproduce a highly complex urban structure. A logarithmic velocity profile with a fluctuating velocity generated using the so-called "Vortex Method" available in Fluent (Mathey et al, 2006) has been used. Through this method, in the inlet plane, a given number $N$ of vortices is generated at each time step. Their intensity and size depends on the imposed local value of the turbulent intensity $I$ (taken as 5\%) and the molecular to turbulent viscosity ratio (taken as 10\%). More details about this technique and validation studies can be found in (Mathey et al, 2006). Based on prior studies that have looked at air flow around a wall-mounted cube, it has been considered that the flow field can be simulated in an accurate way with a vortex number of $N=190$ (Gousseau et al, 2011). The same value has been also utilized in this simulation. The log law mean profile simply follows the classic formulation:

$$
U(z)=\frac{u_{*}}{k}\left[\ln \left(\frac{z d}{z_{0}}\right)\right],
$$

where terrain roughness is set to $z_{0}=0.03 \mathrm{~m}$ (aiming to reproduce the flow features of a highly rough urban environment) and the friction velocity to $u_{*}=0.34 \mathrm{~m} \mathrm{~s}^{-1}$. The resulting profile (see Fig. 5b) yields a very strong shear that is quite distinct from the laminar profile, and the two analysed together thus provide a wide range of possible inflows.

c) Inlet \#3: Has been defined to match (Brown et al, 2000) inlet conditions for validation purposes. The inlet velocity profile obtained from (Brown et al, 2000) is shown in Figure 5 b. It is intermediate between inlets \#1 and \#2 in terms of shear.

Inlet \#3 has only been tested for the flat roof geometry. Inlet \#1 and \#2 on the other hand have been tested for all studied building geometries.

\section{Results and Discussion}

In Figure 7, the numerical experiments (using Inlet\#3) for the flat roof geometry are validated against data from (Brown et al, 2000) who, for a similar setup with an array of 7 buildings, studied the air flow and turbulence statistics. Vertical profiles have been 
compared for canyon \#2 $\left(\mathrm{FC}_{2}, \mathrm{MC}_{2}, \mathrm{BC}_{2}\right)$, canyon for which detailed TKE data for 5 vertical rakes is provided, canyon $\# 6$ ( $\left.\mathrm{FC}_{6}, \mathrm{MC}_{6}, \mathrm{BC}_{6}\right)$ for comparison purposes with the canyon where the LES results have been displayed, and downstream $\left(\mathrm{BC}_{0.1}, \mathrm{BC}_{5.5}\right)$ (see Fig. 3 for locations). The mean velocity results are in very good agreement in canyon \#6 as well as downstream of the array, with differences of about 5\%. The turbulent kinetic energy $\left(T K E=2 / 2=\left(\begin{array}{c}2 \\ u\end{array}+\underset{v}{2}+\underset{w}{2}\right) / 2\right.$, where $\sigma^{2}$ is the total variance $)$ plots display larger discrepancies than the mean velocity results (Fig. 7d); however the differences remain acceptable. Overall, the profile trends are captured accurately and the quantitative errors remain moderate.

The numerical experiments (using Inlet \#1) have also been validated against the wind tunnel results we acquired in this study (only in canyon \#6) as shown in Figure 8. The measurements gathered from the wind tunnel represent the total $U_{t}$ collected with the single hotwire. Since the hotwire was oriented perpendicular to the axis of the approaching flow, it was not sensitive to the cross-stream flow and its measurement thus mainly represent the streamwise and vertical components. Furthermore, the hotwire does not capture the directionality of the approaching flow, so absolute values have been considered for the plot comparisons with the wind tunnel data. $U_{t}$ (total velocity) represents the vectorial magnitude $\left(U^{2}+W^{2}\right)^{1 / 2}$. The total variance, $\sigma_{t}^{2}$ accounts for $\sigma_{u}^{2}+$ $\sigma_{w}{ }^{2}$, and the total root mean square (rms) is thus represented by $\sigma_{t}$. The reference velocity $U_{r}$ has been considered at $2.5 \mathrm{H}$ upstream. We also plot the full flow velocity magnitude $U$ (which will be very close to $U_{t}$ since mean $\mathrm{V} \approx 0$ ) and the full variance $\sigma^{2}$, with contributions from all velocities components $u, v$ and $w$ from the LES results for illustration, although these cannot be directly compared to the measurements. In addition, to capture the errors in the wind tunnel measurements attributed to either incomplete statistical convergence or incomplete homogeneity in the cross-stream direction, we collected data from multiple cross-stream locations and we show the standard deviation between their measurements as error bars on the wind tunnel data in the upcoming plots to delineate the possible range of experimental data. 

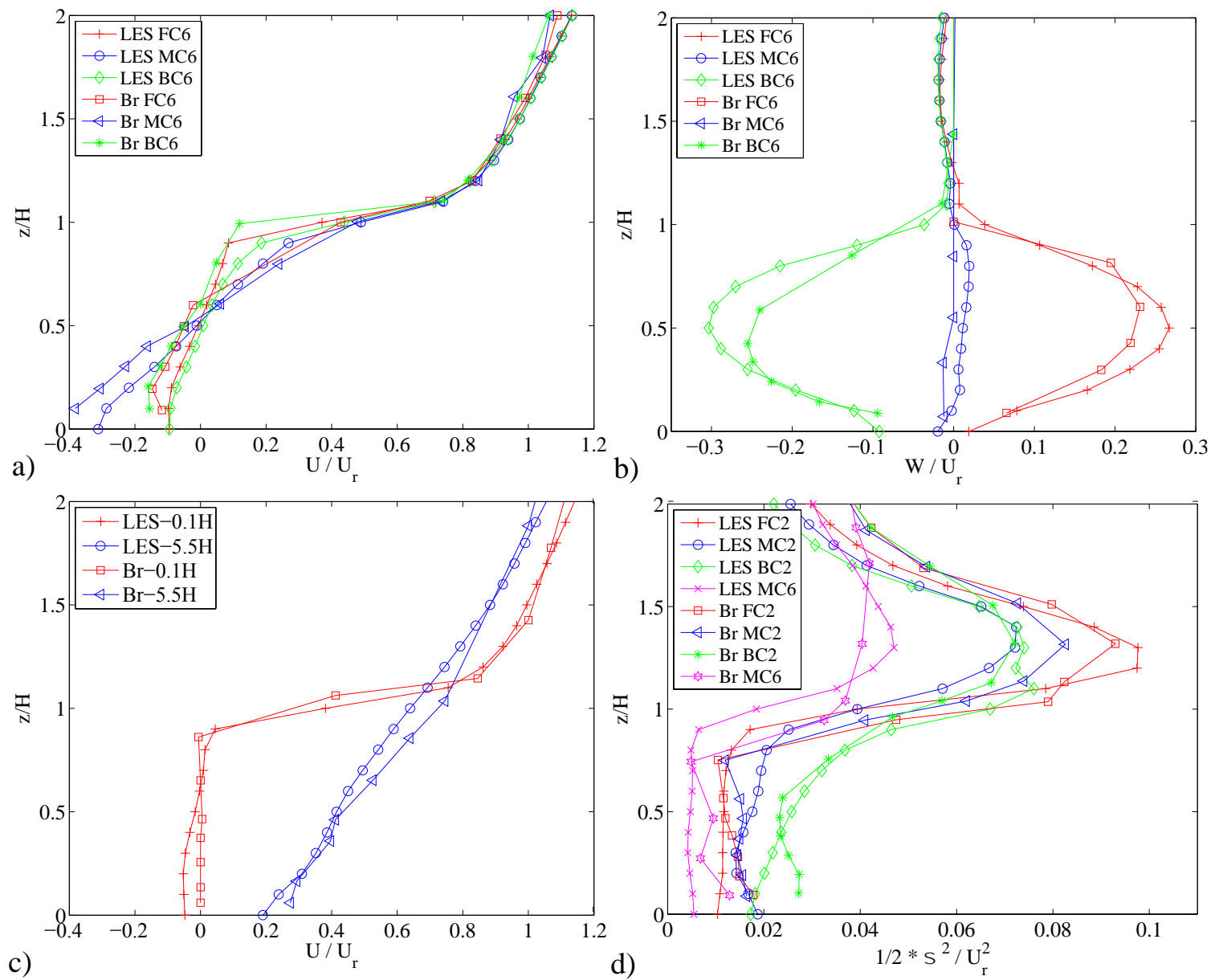

Fig 7 LES flat roof comparison following Inlet \#3 inflow conditions compared with (Brown et al, 2000). As shown in the graphs, LES stands for the results obtained through the large eddy simulations, and $\mathrm{Br}$ stands for the results obtained by (Brown et al, 2000); refer to Figure 3 for location information and acronyms. a) $U$ velocity profile in canyon $\# 6$ (for $\mathrm{FC}_{6}, \mathrm{MC}_{6}, \mathrm{BC}_{6}$ ). b) $W$ velocity profile for canyon \#6 (for $\mathrm{FC}_{6}, \mathrm{MC}_{6}, \mathrm{BC}_{6}$ ). c) $U$ velocity profile for downstream $\mathrm{B} 0.1 \mathrm{H}, \mathrm{B} 5.5 \mathrm{H}$. d) TKE profiles for canyon \#2 (for $\mathrm{FC}_{2}, \mathrm{MC}_{2}, \mathrm{BC}_{2}$ ) and for canyon \#6 (for MC6). All velocities are normalized using the velocity $U_{r}$ at $2.5 \mathrm{H}$ upstream.

Three measuring lines $\left(\mathrm{FC}_{6}, \mathrm{MC}_{6}, \mathrm{BC}_{6}\right)$ have been gathered for canyon \#6, for each geometric configuration (see Fig. 3). The comparison of the wind tunnel and LES results for the flat roof geometry show a good agreement with largest differences at elevations between $1 \mathrm{H}$ and $2 \mathrm{H}$ for the mean velocities (compare black lines and markers in Fig. 8a). The $\sigma_{t}^{2}$ plots show a good agreement with differences smaller than 15\% (compare black lines and markers in Fig. 8b). The comparison of the mean velocity between the wind tunnel and LES results for the pitched roof geometry also show a very good agreement with differences smaller than 5\% (see Fig. 8c); similarly good agreement is observed for the case of the round roof geometry mean velocity (see Fig. 8d). On the other hand, the 
comparison for the $\sigma_{t}^{2}$ plots between the wind tunnel and LES results show larger differences for both the pitched and round roof geometries, compared to the $\sigma_{t}^{2}$ plots of the flat roofs or to the means. While the wind tunnel and LES results agree qualitatively on higher turbulence levels for the pitched and round roofs in comparison to the flat roof, LES appears to overestimate the turbulence statistics in comparison to the wind tunnel results. The mismatch in the inlet velocity or an underestimation by the hot wire of the variance due to the low velocity in the canyon might be potential causes of this discrepancy; but one would expect such a mismatch to also influence the results of the flat roof and the means. This leaves us with the distinct possibility that LES has more difficulty in capturing the turbulence inside the canopy over non-flat roofs, and this is affecting turbulence levels inside as well as outside the canopy layer. An additional challenge for LES over the round and pitched roofs is to capture the separation correctly ((Temmerman et al, 2003) for an in-depth analysis of this challenge for LES over a round hill). For the flat roof, the separation occurs at the corner of the building (easy to capture) (Aynsley, 1999), but for the other roofs, a clear separation point is not imposed by the geometry and the LES might have more difficulty matching it exactly given that, particularly in simulations like ours, LES relies on wall-modelling. In addition, the separation will be sensitive to the effective $R e$ of the simulation. 

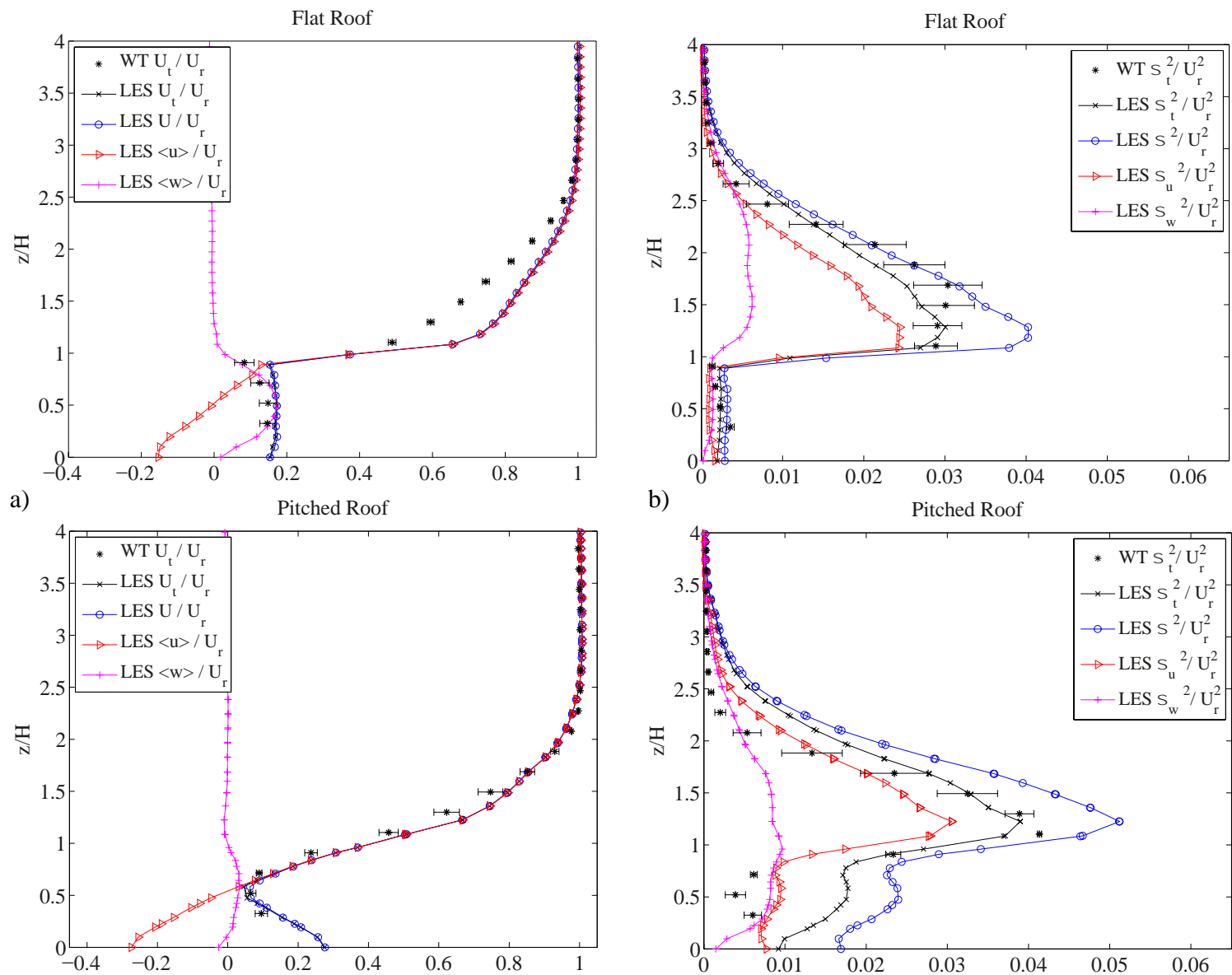

b)

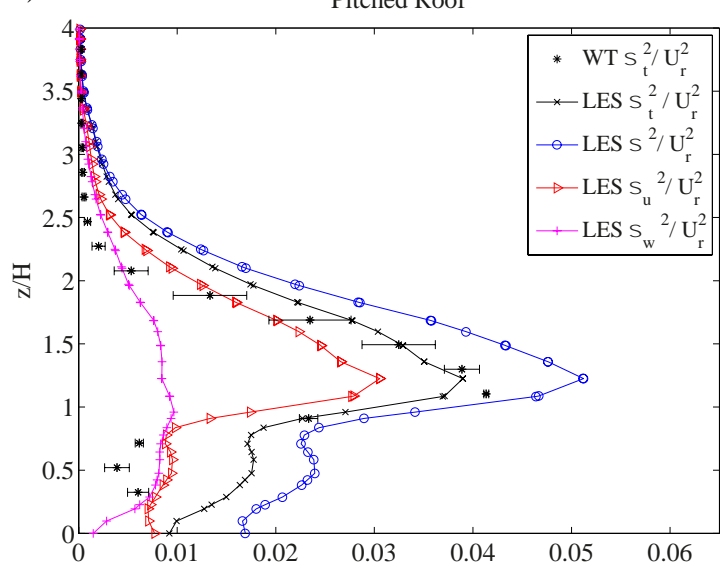

c)

Round Roof

d)
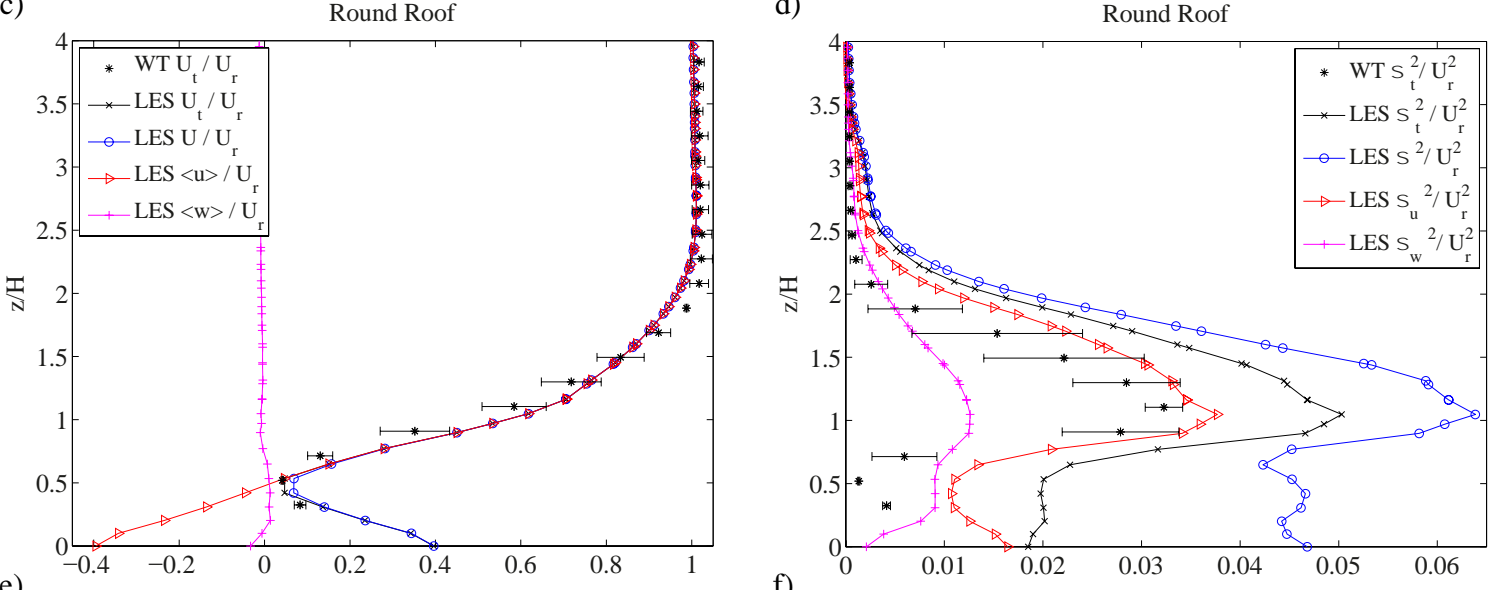

f)

Fig 8 Wind Tunnel and LES result (with imposed Inlet \#1 inflow conditions) comparison for canyon \#6 until $4 H$. All values are normalized by $U_{r}$. The left panels depict the wind tunnel $U_{t}$ and LES $U_{t}, U,\langle u\rangle$ and $\langle w\rangle$ for a) Flat roof, c) Pitched roof, and e) Round roof. The right panels depict the wind tunnel $\sigma_{t}^{2}$ and LES $\sigma_{t}^{2}, \sigma^{2}, \sigma_{u}^{2}, \sigma_{w}^{2}$ for b) Flat roof, d) Pitched roof, and f) Round roof. For validation, the total components plotted in black should be compared. 
Aiming to understand the effect of $R e$ on simulations of flow over smooth geometries, for the round and the flat roof geometries, further simulations have been performed at higher $R e$ (corresponding to a real world prototype). The results at the higher $R e$ are presented in the Appendix; but will be compared in the text to the results at the lower $R e$, model. Figure 9 depicts a vector plot of the mean velocity overlaid on a pseudo-colour plots of the sum of the streamwise and vertical velocity standard deviation at the model Reynolds number for all geometries, while the plots shown in Figure A1 are the same but based on the real prototype $R e$ and for the flat roof and round roofs only. The results obtained for the flat roof geometry, qualitatively speaking, show reasonably consistent features between the results at both Reynolds numbers. Recirculation within canyon is observed in both cases and the turbulence intensity appears consistently highest in the shear layer near the building roofs in both simulations. The round roof geometry, on the other hand, appears to be more sensitive to the $R e$. The separation point on the leeward face of the upwind building and the reattachment point on the windward face of the downwind building are both shifted at higher $R e$; the flow velocity in the shear layer is increased, weakening the within canyon vortex. Therefore, it appears reasonable to infer that larger differences are observed for the round roof geometry as $R e$ varies. The differences observed between the wind tunnel and LES variances in Figure 8f might hence be attributed to the two inter-related factors: (1) the sensitivity of round roof geometries to the Reynolds number, and (2) the difficulty wall-modelled LES faces in capturing how $R e$ exactly affects the location of the separation and reattachment points. This finding is consequential; it implies that validating an LES code for flat roofs or sharp geometries in general does not guarantee equivalent performance for all geometries and further studies are needed to fully investigate this topic. Solving this challenge is beyond the scope of this paper since it requires advances in wall-modelling of LES that remain lacking, but these comparisons serve to inform us on the need for these advances and on the uncertainty in our simulation results. 

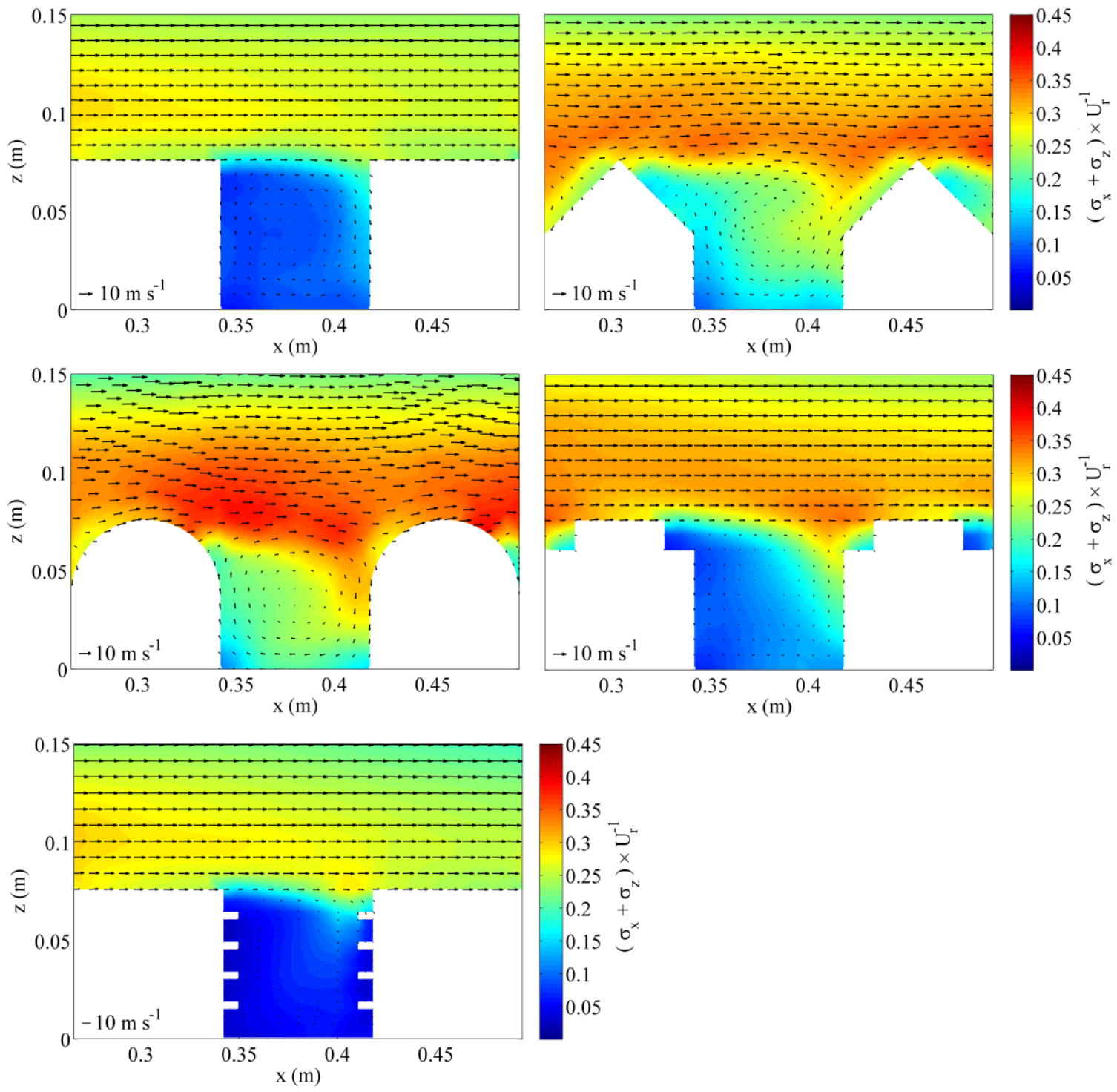

Fig 9 LES results (with imposed Inlet \#1 inflow conditions) for air flow and turbulence statistics for the 5 different building geometries. The plots show $\sigma_{t}$ as a pseudo-colours, and the mean velocity as a vector plot.

Despite the potential inaccuracy in the LES and the need to consider the influence of $R e$, the experiments and the simulations at all $R e$ unambiguously show significant differences in the flow and turbulence fields between the various roof geometries. This can be seen qualitatively in Figure 9. To better quantify these differences, we depict the turbulence statistics profiles from the different geometries on the same plot in Figure 10, 
from both the experiment and the simulations. Within canyon, the pitched and round roofs show larger mean velocity gradients indicating a stronger circulation than in the case of the flat roof (Fig. 10a), and a higher potential for turbulence generation. While the round roof shows largest velocity gradients, the differences in the mean velocities between the pitched and round roofs are more subtle than those observed in comparison to the flat roof. These differences are in agreement with the fog studies that also display large differences between the flat and the two other roof geometries, and more similar trends between the pitched and round roofs (Fig. 4). The velocity variances within canyon, show similar tendencies (Fig. 10b). The LES results feature large differences between the studied geometries with differences of an order of magnitude between the flat and the other two roof geometries. The wind tunnel results, while also showing larger turbulence levels for the case of the pitched and round roofs in comparison to the flat roof, indicate smaller differences than those observed in the LES calculations.
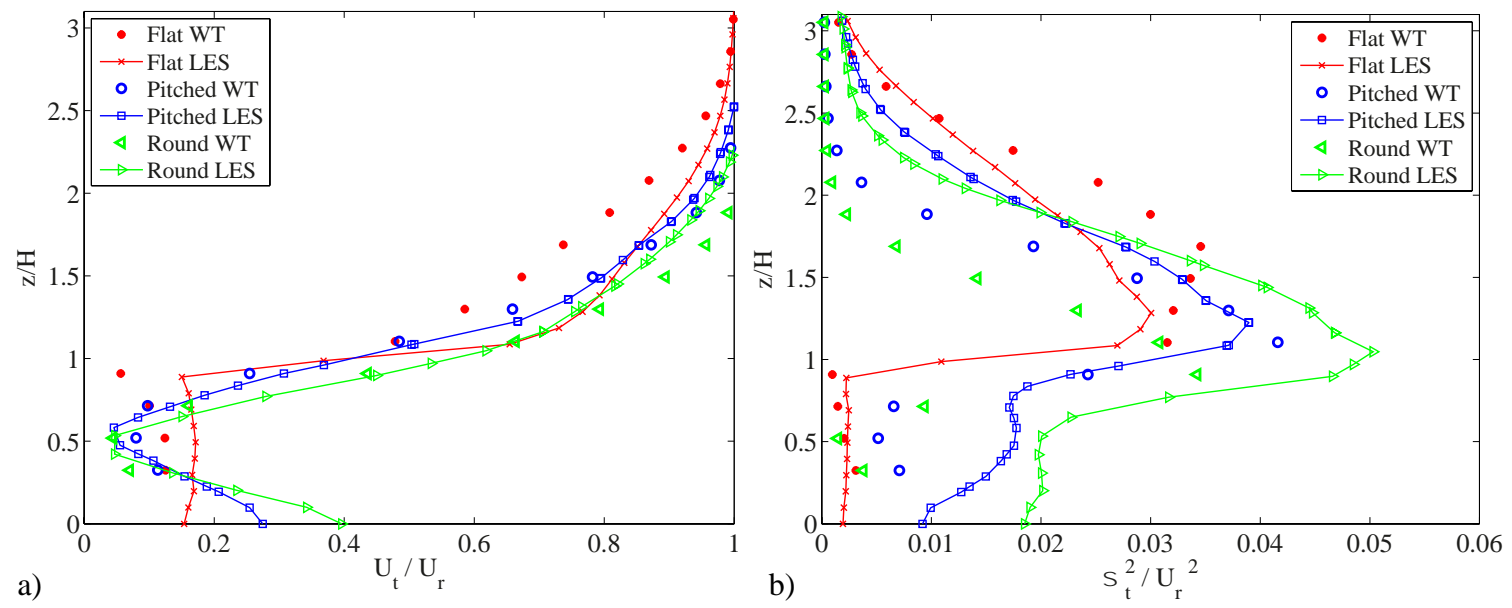

Fig 10 Overlay of wind tunnel and LES result (with imposed Inlet \#1 inflow conditions) for canyon \#6 up to a height of $3 H$ for the Flat, Pitched and Round roof geometries. a) wind tunnel $U_{t}$ and LES $U_{t}$, b) wind tunnel $\sigma_{t}^{2}$ and LES $\sigma_{t}^{2}$.

A comparison of the LES flow patterns in the central vertical plane of canyon \#6 for the 3 different roof geometries, in addition to the two facade configurations that were not featured in the wind tunnel, is shown in Figures 9, 11, and 12. These plots include LES results using Inlet \#1. The mean velocity vector $\vec{U}_{t}=\vec{U}+\vec{W}$ is presented as a vector plot. 
The pseudo-colour plots display information about the root mean square $\sigma_{t}$, absolute pressure, and cross-stream vorticity.

As with roof geometries, the variations in façade configurations seem to have a strong impact on the turbulence statistics. The results corroborate the ones depicted in the previous figures, but spatial features in the $x-z$ plane provide richer information on the influence of roof and façade features on the flow. The magnitude of the turbulence intensity increases for the pitched and round roof geometries in comparison to the flat roof, the round roof being the geometry where the largest turbulence intensities are observed. The presence of balconies decreases the turbulence intensity in the street canyon. While the terrace appears to create a weaker effect, it increases the depth of the shear layer in comparison with the flat roof geometry. In all cases, the highest turbulence levels are observed in the shear zone near the roof level, but the depth of this zone varies for the different cases. While the shear zone penetrates the canyon in all studied cases in the area adjacent to the upstream face, the turbulence intensity and the turbulent exchanges between the street canyon and the air above seem to differ with the different geometries. For the pitched and round roofs, the flow redirection that takes place on the upstream roof slope creates a strong downdraft over that face, inducing higher turbulent intensities and potentially enhancing street ventilation and pollutant dispersion.

Within canyon velocity standard deviations $\left(\sigma_{t}\right)$ for the pitched and round roofs are more than double those observed for the flat roof geometry (see Fig. 9). These differences appear to be mostly related to variations in $\sigma_{w}$, which displays large differences between the studied geometries than $\sigma_{u}$. The LES results show that the round roof geometry generates the largest $\sigma_{w}$, while the flat roof reduces the vertical turbulent exchanges between the urban canopy layer and the free atmosphere. The terraced building, in comparison to the flat roof, increases air flow and turbulence within canyon. On the other hand, the presence of balconies affects the stagnation of air within canyon considerably, diminishing the strength of the mean recirculating flow and reducing the turbulence intensity.

The pressure differences obtained from LES for the flat roof remain about $\Delta P=20$ $\mathrm{Pa}$, increasing to $30 \mathrm{~Pa}$ for the pitched roof and to $40 \mathrm{~Pa}$ for the round roof configurations (see Fig. 11). For the flat roof, the stagnation point is observable in the windward vertex 
of the roof corner, while for the pitched and round roofs, it is located in the middle of the roof slopes. The flow separation point is at the leeward vertex for the flat roof, slightly below the crest for the pitched roof, and almost half way down the roof height for the round geometry. The terrace building and balcony buildings, present analogous pressure differences to those observed for the flat roof. These differences in the separation and stagnation points explain the dissimilarities in the velocity profiles, as well as the distinct cross-stream vorticity maps shown in Figure 12 . The vorticity patterns are more complex for the pitched and round roofs, and high vorticity levels span a larger height range around the roof top compared to the other geometries, again indicating stronger turbulence and mixing. Note that both the vector plots of the velocity and pseudo-colour plot of vorticity are from an instantaneous field in Figure 12 (rather than from means as in Fig. 9 and Fig. 11) 

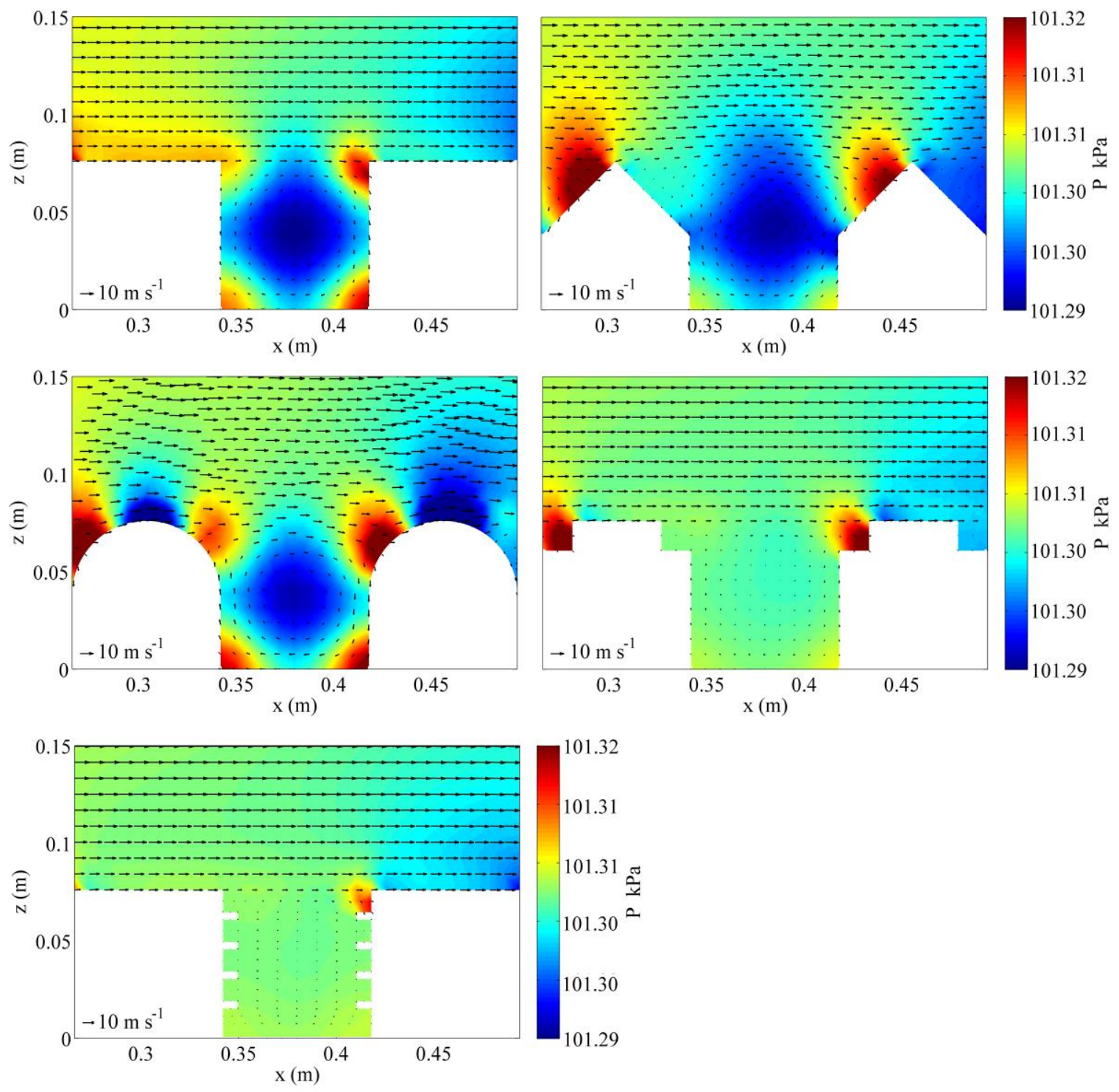

Fig 11 LES results (with imposed Inlet \#1 inflow conditions) for air flow and mean pressure for the 5 different building geometries. Mean velocity is displayed with vectors. The pseudo-colours show the mean pressure plots. 

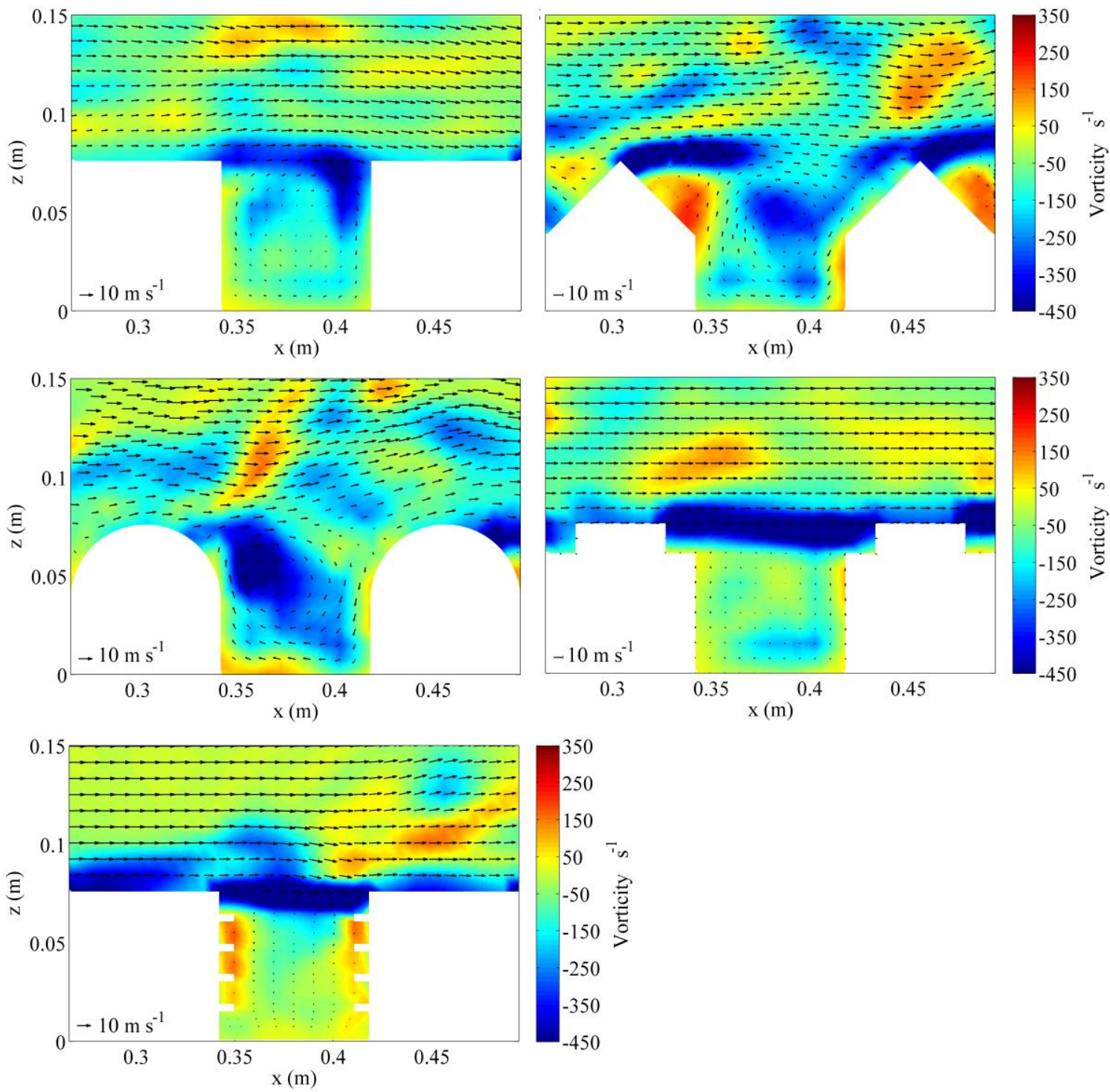

Fig 12 LES results (with imposed Inlet \#1 inflow conditions) for the instantaneous cross-stream vorticity for the 5 different building geometries. Instantaneous velocity $\vec{U}+\vec{W}$ is displayed with vectors. The pseudo-colours show mean $y$-vorticity plots.

An important aspect to consider is the upstream buildings configuration, which dictates inflow conditions for downstream buildings. In Figure 9 the differences created by the upstream geometries on the incoming flows can be observed. The highest turbulence intensities in the incoming flow are observed for the round and pitched roof geometries followed by the building with terraces. The flat roof and building with balconies show the lowest turbulence intensities. As such, and despite the fact that in Figure 1 we have observed that the flow after canyon \#4 is less dependent on the inflow 
to the domain, the upstream geometries play a decisive role in the turbulence characteristics of the incoming flow for a given canyon. Therefore, configurations with mixed roof geometries are going to behave very differently from canopies with homogeneous topographies.

The qualitative fog analysis experiments (see Fig. 4) and the LES instantaneous velocity plots shown in Figures 6 and 12 further corroborate the differences between the studied 3 roof geometries. The mean recirculating flow develops more clearly for the flat roof geometry, inducing a stronger trapping of the air volume within the street canyon (see Fig. 4). For the geometries of the pitched and round roofs, less organized turbulent structures dominate the air flow characteristics, inducing larger exchanges between the street canyon and free atmosphere. In the case of the round geometry, an elevated small vortex is developed at the upstream face that appears to relate to the position of the stagnation point in the roof as shown in the numerical simulations. In the centre of the street canyon, the flat roof geometry displays low velocities that suggest the presence of the within-canyon vortex. The pitched roof results show less organized flow structures, and overall higher velocities are observed within the canyon suggesting larger exchanges with the over canopy flows. The round roof geometry results also show less organized flow features within canyon, while as observed in the fog studies, they display a roof-level vortex attached to the upstream slope.

\section{Conclusions}

Through wind tunnel experiments and large eddy simulations, different building geometries have been studied to produce a comparative overview of the impact of some of the most common building typologies on air flow and turbulence statistics in the urban street canyon, as well as to assess the ability of LES to reproduce these impacts. The Fluent LES code for complex architectural geometries was evaluated against wind tunnel experiments. Comparisons demonstrate that the LES results are overall in good agreement with the wind tunnel experiments, and can be used as a basis for understanding the detailed flow dynamics through the time-varying three-dimensional fields they provide. However, the error is found to depend on geometry, with round and pitched roofs exhibiting a larger discrepancy due to the fact that replicating the exact 
location of the separation point is more difficult with these geometries. Moreover, a higher sensitivity of the separation and reattachment points locations to the Reynolds number is documented for these geometries, underlining the more challenging flow attributes that LES has to capture. Therefore, while the LES with wall-modelling used here provides results that are qualitatively reliable and consistent with wind tunnel data, more research is needed on the impact of geometry on the performance of wall-models. Once the numerical model had been evaluated against the wind tunnel results collected for this study, two more inflow conditions were tested for validation purposes with prior literature (Brown et al, 2000), as well as to reproduce inflow characteristics more similar to real urban environments.

The results indicated that the influence of the inflow characteristics (as well as the flow blockage due to finite domain height) are less perceivable by canyon \#6 compared to canyons \#1 to \#4. The influence of smaller architectural features in the upstream building geometries has also been discussed to conclude that not only urban scale considerations, but architectural scale features such as building roof geometries and balconies should be taken into account when setting up the upstream building conditions given their impact on the turbulence intensity of the incoming flow.

The present results have also shown that architectural features, such as roof geometries or façade elements, play a decisive role in the airflow and turbulence statistics of the urban street canyon. By altering the surface roughness of the interface between the urban canopy layer and the air above, building geometries affect the flow features and pressure gradients over and within the urban street canyons, and as a consequence influence the mean and turbulent exchanges at the pedestrian level. Roofs of complex shapes (round or pitched) result in much higher turbulence intensity and mixing compared to flat roofs, while façade elements such as balconies produce the opposite effect. Therefore, it appears reasonable to suggest that through a formal exploration of building geometries, the ventilation potential of urban canyons can be increased to improve the air quality within the street canyon by reducing the presence of pollutant concentrations and heat entrapment. However, the parameter space of such investigations should also consider the wind angle and the highly-variable large-scale flow patterns that 
are typical in real-cities, but not reproducible in wind tunnel or idealized simulations. The flow patterns we observe here will be sensitive to these two parameters.

Further research is required to study the influence of additional architectural features and the extrapolation of street canyon air flow and turbulence fields obtained from experimental work to real world scenarios, particularly given the sensitivity to the Reynolds number that non-flat roofs displayed in our analyses. This will be the focus of a future publication, which will further elaborate on the results obtained using realistic inflow conditions, and focus on the influence of architecture on the street canyon ventilation potential and air exchange rate.

\section{Acknowledgements}

The authors thank the National Oceanographic and Atmospheric Administration's (NOAA) via the Cooperative Institute for Climate Science (CICS) of Princeton University and the Chair of Structural Design of the Institute of Technology and Architecture, ETH Zurich for their financial support. The help of Tristen Hohman, Dr. Anand Ashok, Mike Vocaturo and Joe Vocaturo was instrumental in the success of the wind tunnel experiments. Elie Bou-Zeid is supported by the US National Science Foundation's Sustainability Research Network Cooperative Agreement 1444758. 


\section{Appendix 1}

In (Figs. A1, A2 and A3), the results for $\sigma$, the mean pressure plots, and the $y$-axis vorticity obtained from the simulations performed at the real prototype $R e$ for two of the studied geometries (the flat roof and the round roof geometries) are displayed. The simulations have been performed using inlet \#1 conditions, that is, a laminar and uniform inflow has been imposed with an inlet velocity of $10 \mathrm{~m} \mathrm{~s}^{-1}$. The results show that the sensitivity to the Reynolds number varies depending on the building geometry. The results obtained for the flat roof geometry, qualitatively speaking, show reasonably consistent features at both the model and prototype $R e$. A within-canyon vortex is observed in both cases and the turbulence intensity appears consistently highest in the shear layer in both simulations. The results obtained for the round roof geometry show a larger $R e$ sensitivity. A delay in the separation point is observed at higher $R e$ (see Fig. A2), and the location of the reattachment point on the downwind building also appears to be sensitive to $R e$. For both geometries, the presence of the within canyon vortex is weakened for higher $R e$ simulations, but this is more clearly perceived in the results obtained for the round roof geometry. Also visible in Figure A2, the negative pressures exerted over building array \#7 (induced due to the lack of further downstream canyons after canyon \#6) are more noticeable for the simulations at real prototype $R e$.
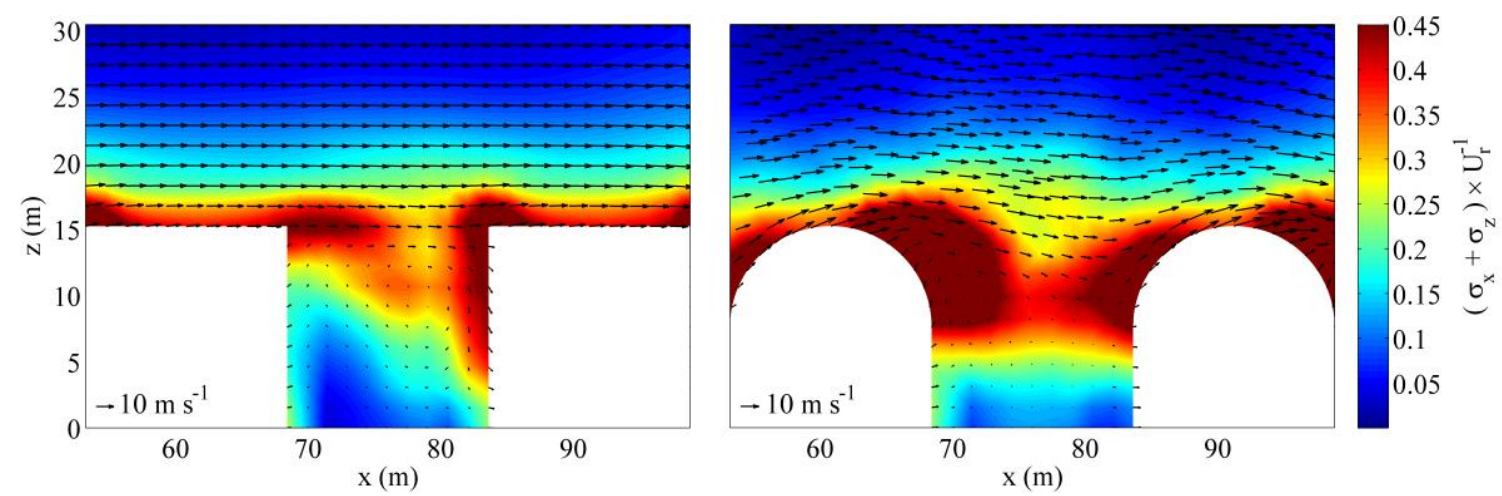

Fig A1 LES results for Reynolds number equivalent to real prototype (with imposed Inlet \#1 inflow conditions) for air flow and turbulence statistics for the round roof and flat roof building geometries. The plots show $\sigma$ as pseudo-colours, and the mean velocity as a vector plot. 
The $y$-vorticity plot (Fig. A3) for the flat roofs show qualitatively consistent patterns for both studied Reynolds numbers. The higher Reynolds number simulations developed for the round roof geometries, on the other hand, display a within canyon vortex located at the centre height of the roofs, instead of the centre of the street canyon as with the model $R e$ simulations. The vorticity plots are however from instantaneous fields, and one can notice the much stronger differences between the instantaneous and mean fields for the round roofs (compare vector plots in the right panels of Figs A1 and A3) than for the flat roofs (compare vector plots in the lefts panels of Figs A1 and A3).

Overall, the main features observed in the model Reynolds number simulations remain in agreement with the higher Reynolds number studies. Higher turbulent intensities are observed for the round roof geometry than for the flat roof geometry; confirming that the flow characteristics within the street canyon are very sensitive to the building geometries.
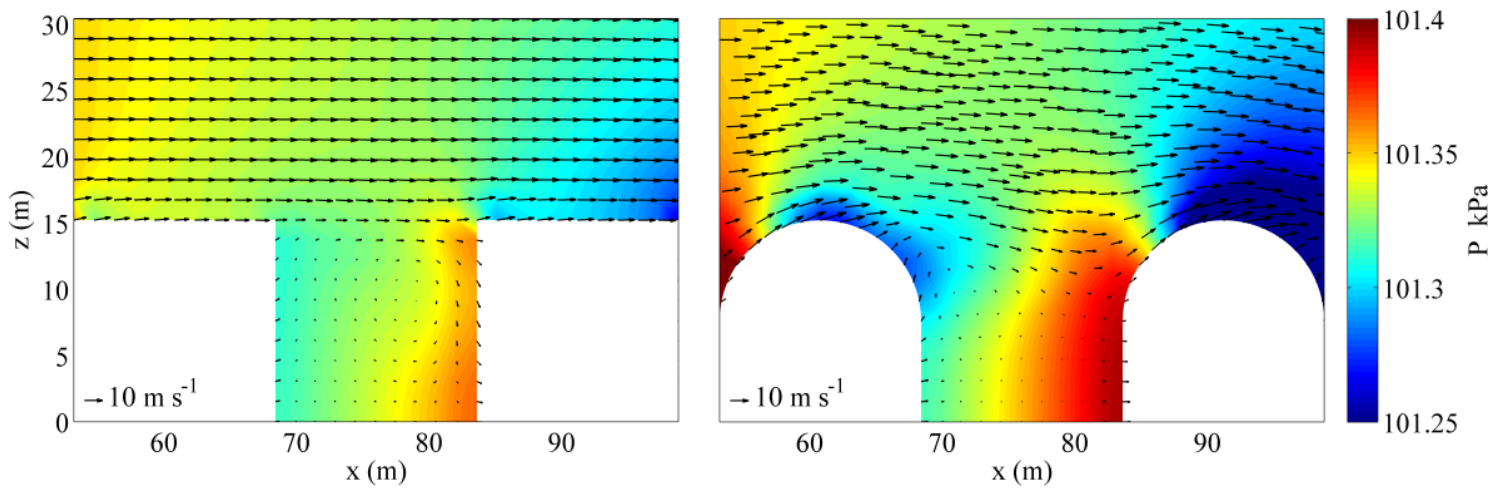

Fig A2 LES results for Reynolds number equivalent to real prototype (with imposed Inlet \#1 inflow conditions) for air flow and turbulence statistics for the round roof and flat roof building geometries. Mean velocity is displayed with vectors. The pseudo-colours show the mean pressure plots.
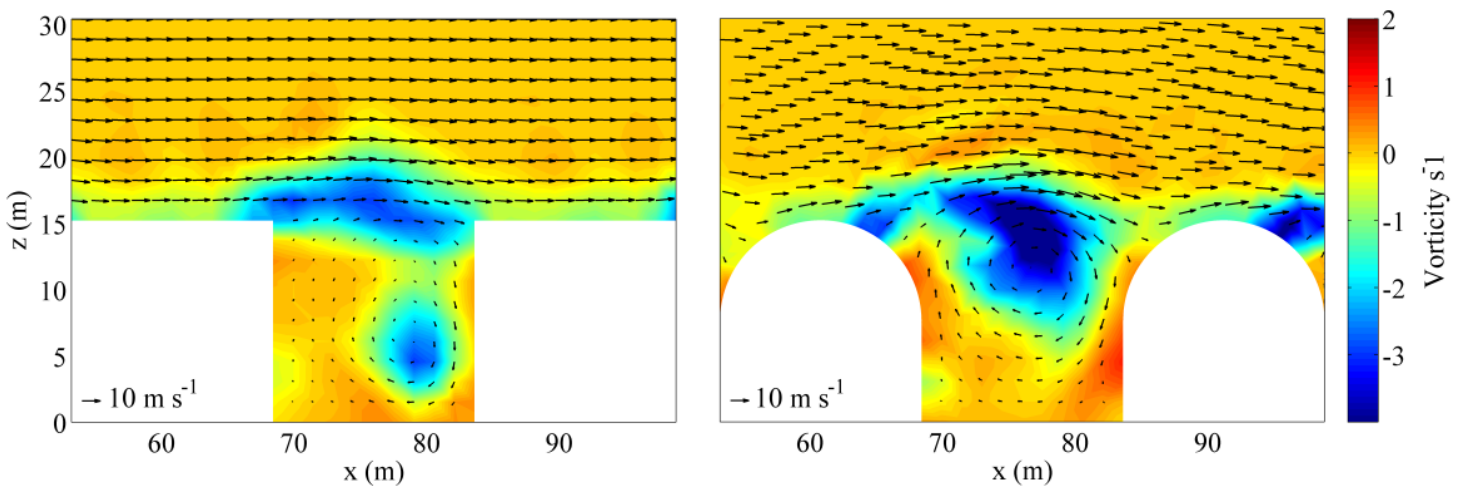

Fig A3 LES results for Reynolds number equivalent to real prototype (with imposed Inlet \#1 inflow conditions) for air flow and turbulence statistics for the round roof and flat roof building geometries. Instantaneous velocity $\vec{U}+\vec{W}$ is displayed with vectors. The pseudo-colours show mean $y$-vorticity plots. 


\section{References}

ANSYS, 2013. ANSYS Fluent Theory Guide (Report). Release 15.0. Ansys, Inc., Canonsburg, PA.

Assimakopoulos, V.D., ApSimon, H.M., Moussiopoulos, N., 2003. A Numerical Study of Atmospheric Pollutant Dispersion in Different Two-Dimensional Street Canyon Configurations. Atmos Environ 37, 4037-4049.

Aynsley, R.M., 1999. Shape and Flow: The Essence of Architectural Aerodynamics. Archit Sci Rev 42, 69-74.

Baik, J.J., Park, R.S., Chun, H.Y., Kim, J.J., 1999. A Laboratory Model of Urban StreetCanyon Flows. J Appl Meteorol 39, 1592-1600.

Berkowicz, R., 2001. The European Research Network TRAPOS -Results and Achievements, Optimisation of Modelling Methods for Traffic Pollution in Streets.

Blackman, K., Perret, L., Savory, E., 2014. Effect of Upstream Flow Regime on Street Canyon Flow Mean Turbulence Statistics. Environ Fluid Mech 15, 823-849.

Bou-Zeid, E., 2005. Large Eddy Simulation of Atmospheric Boundary Layer Flow over Heterogeneous Surfaces. Johns Hopkins University, Baltimore, Maryland.

Bou-Zeid, E., Overney, J., Rogers, B.D., Parlange, M.B., 2009. The Effects of Building Representation and Clustering in Large-Eddy Simulations of Flows in Urban Canopies. Boundary-Layer Meteorol 132, 415-436.

Britter, R.E., Hanna, S.R., 2003. Flow and Dispersion in Urban Areas. Annu Rev Fluid Mech 35, 469-496.

Brown, M.J., Lawson, R.E., DeCroix, D.S., Lee, R.L., 2000. Mean Flow and Turbulence Measurements around a 2-D Array of Buildings in a Wind Tunnel, Conf. Appl. of Air Pollut. Meteorol.,11th. Am Meteorol Soc, Long Beach, Calif.

Counehan, J., 1971. Wind Tunnel Determination of the Roughness Length as a Function of the Fetch and the Roughness Density of Three-dimensional Roughness Elements. Atmos Environ 5, 637-642.

Courant, R., Friedrichs, K., Lewy, H., 1928. On the Partial Difference Equations of Mathematical Physics. Mathematische Annalen \& IBM J Res and Dev 11, 215-234.

Dezső-Weidinger, G., Stitou, A., Van Beeck, J., Riethmuller, M.L., 2003. Measurement of the Turbulent Mass Flux with PTV in a Street Canyon. J Wind Eng Ind Aerodyn 91, 1117-1131.

Fernando, H.J.S., 2010. Fluid Dynamics of Urban Atmospheres in Complex Terrain. Annu Rev Fluid Mech 42, 365-389.

Franke, J., Hellsten, A., Schlunzen, H., Carissimo, B., 2007. COST Action 732 Best Practice Guideline for CFD Simulation of Flows in the Urban Environment, Quality Assurance and Improvement of Microscale Metereological Models. University of Hamburg, Hamburg.

Georgiadis, N.J., Rizzetta, D.P., Fureby, C., 2010. Large-Eddy Simulation: Current Capabilities, Recommended Practices, and Future Research. AIAA Journal 48, 17721784.

Gousseau, P., Blocken, B., Stathopoulos, T., van Heijst, G.J.F., 2011. CFD Simulation of Near-Field Pollutant Dispersion on a High Resolution Grid: a Case Study by LES and RANS for a Building Group in Downtown Montreal. Atmos Environ 45, 428-438. 
Grimmond, C.S.B., King, T.S., Roth, M., Oke, T.R., 1998. Aerodynamic Roughness of Urban Areas Derived From Wind Observations Boundary-Layer Meteorol 89, 1-24. Hosker, R., 1987. The Effects of Buildings on Local Dispersion, Modeling the Urban Boundary Layer. Am Meteorol Soc, 95-159.

Huang, Y., Hu, X., Zeng, N., 2009. Impact of Wedge-Shaped Roofs on Airflow and Pollutant Dispersion Inside Urban Street Canyons. Build and Environ 44, 2335-2347. Huang, Y.D., He, W.R., Kim, C.N., 2014. Impacts of Shape and Height of Upstream Roof on Airflow and Pollutant Dispersion inside an Urban Street Canyon. Environ Sci Pollut Res 22, 2117-2137.

Hunter, L.J., Watson, I.D., Johnson, G.T., 1990. Modelling Air Flow Regimes in Urban Canyons. Energy and Build 15-16, 315-324.

Inagaki, A., Castillo, M.C.L., Yamashita, Y., Kanda, M., Takimoto, H., 2012. Large-Eddy Simulation of Coherent Flow Structures within a Cubical Canopy. Boundary-Layer Meteorol 142, 207-222.

Jimenez, J.M., 2007. High Reynolds Number Flows about Bodies of. Princeton University, Princeton.

Kastner-Klein, P., Berkowicz, R., Britter, R., 2004. The Influence of Street Architecture on Flow and Dispersion in Street Canyons. Meteorol Atmos Phys 87, 121-131.

Kastner-Klein, P., Plate, E.J., 1999. Wind-Tunnel Study of Concentration Fields in Street Canyons. Atmos Environ 33, 3973-3979.

Kellnerova, R., Kukacka, L., Jurcakova, K., Uruba, V., Janour, Z., 2012. PIV Measurement of Turbulent Flow Within a Street Canyon: Detection of Coherent Motion. J Wind Eng Ind Aerodyn 104-106, 302-313.

Kovar-Panskus, A., Louka, P., Sini, J.F., Savory, E., Czech, M., Abdelquari, A., Mestayer, P.G., Toy, N., 2002. Influence of Geometry on the Mean Flow within Urban Street Canyons-A Comparison of Wind Tunnel Experiments and Numerical Simulations. Water Air Soil Pollut 2, 365-380.

Li, D., Bou-Zeid, E., 2013. Synergistic Interactions between Urban Heat Islands and Heat Waves: The Impact in Cities Is Larger than the Sum of Its Parts. J Appl Meteorol Clim 52, 2051-2064.

Li, D., Bou-Zeid, E., Oppenheimer, M., 2014. The Effectiveness of Cool and Green Roofs as Urban Heat Island Mitigation Strategies. Environ Res Lett 9, 055002.

Ligrani, P.M., Bradshaw, P., 1987. Spatial resolution and measurement of turbulence in the viscous sublayer using subminiature hot-wire probes Exp Fluids 5, 407-417.

Liu, C.H., Barth, M.C., Leung, D.Y.C., 2004. Large-Eddy Simulation of Flow and Pollutant Transport in Street Canyons of Different Building-Height-to-Street-Width Ratios. J Appl Meteorol 43, 1410-1423.

Mathey, F., Cokljat, D., Bertoglio, J.P., Sergent, E., 2006. Assessment of the Vortex Method for Large Eddy Simulation Inlet Conditions. Progress in Comp Fluid Mech 6, 58-67.

Oke, T.R., 1973. City Size and the Urban Heat Island. Atmos Environ 7, 769-779.

Oke, T.R., 1988. The Urban Energy Balance. Progress in Phys Geogr 12, 471-508.

Piomelli, U., Moin, P., Ferziger, J.H., 1988. Model Consistency in Large Eddy Simulation of Turbulent Channel Flows. Phys Fluids 31, 1884-1891.

Pope, S.B., 2000. Turbulent flows. Cambridge University Press, New York. 
Rafailidis, S., 1997. Influence of Building Areal Density and Roof Shape on the Wind Characteristics Above a Town. Boundary-Layer Meteorol 85, 255-271.

Rafailidis, S., Schatzmann, M., 1996. Physical Modeling of Car Exhaust Dispersion in Urban Street Canyons. The Effect of Slanted Roofs. Air Pollut Model Appl XI.

Raupach, M.R., Thom, A.S., Edwards, I., 1980. A Wind Tunnel Study of Turbulent Flow Close to Regularly Arrayed Rough Surfaces. Boundary-Layer Meteorol 18, 373397.

Salizzoni, P., Marro, M., Soulhac, L., Grosjean, N., Perkins, R.J., 2011. Turbulent Transfer Between Street Canyons and the Overlying Atmospheric Boundary Layer. Boundary Layer Metereol 141, 393-414.

Salizzoni, P., Soulhac, L., Mejean, P., Perkins, R.J., 2008. Influence of a Two-scale Surface Roughness on a Neutral Turbulent Boundary Layer. Boundary-Layer Meteorol 127, 97-110.

Savory, E., Perret, L., Rivet, C., 2013. Modelling Considerations for Examining the Mean and Unsteady Flow in a Simple Urban-Type Street Canyon. Meteorol Atmos Phys 121, 1-16.

Shur, M.L., Spalart, P.R., Strelets, M.K., Travin, A.K., 2008. A hybrid RANS-LES approach with delayed-DES and wall-modelled LES capabilities. Int J Heat Fluid Flow 29, 1638-1649.

Sini, J.F., Anquetin, S., Mestayer, P.G., 1996. Pollutant Dispersion and Thermal Effects in Urban Street Canyons. Atmos Environ 30, 2659-2677.

Smagorinsky, J., 1963. General Circulation Experiments with the Primitive Equations, in: James E.Caskey, J. (Ed.), Monthly Weather Review. Weather Bureau, Washington D.C.

Takano, Y., Moonen, P., 2013. On the Influence of Roof Shape on Flow and Dispersion in an Urban Street Canyon. J Wind Eng Ind Aerodyn 123, 107-120.

Temmerman, L., Leschziner, M.A., Mellen, C., Fröhlich, J., 2003. Investigation of Subgrid-Scale Models and Wall-Function Approximations in Large Eddy Simulation of Separated flow in a Channel with Streamwise Periodic Constrictions. Int J Heat Fluid Flow 24, 157-180.

Theodoridis, G., Moussiopoulos, N., 2000. Influence of Building Density and Roof Shape on the Wind and Dispersion Characteristics in an Urban Area. A numerical study. Environ Monit Assess 65, 407-415,.

WHO, 2014. World Urbanization Prospects 2014. United Nations.

Xie, X., Huang, Z., Wang, J.S., 2005. Impact of Building Configuration on Air Quality in Street Canyon. Atmos Environ 39, 4519-4530.

Xie, Z.T., Castro, I.P., 2009. Large-Eddy Simulation for Flow and Dispersion in Urban Streets. Atmos Environ 43, 2174-2185.

Yaghoobian, N., Kleissl, J., Paw, U.K.T., 2014. An Improved Three-Dimensional Simulation of the Diurnally Varying Street-Canyon Flow. Boundary-Layer Meteorol 153, 251-276.

Yamartino, R.J., Wiegand, G., 1986. Development and Evaluation of Simple Models for the Flow, Turbulence and Pollutant Concentration Fields within an Urban Street Canyon. Atmos Environ 20, 2137-2156.

Yassin, M.F., 2011. Impact of Height and Shape of Building Roof on Air Quality in Urban Street Canyons. Atmos Environ 45, 5220-5229. 
Zajic, D., Fernando, H.J.S., Calhoun, R., Princevac, M., Brown, M.J., Pardyjak, E.R., 2011. Flow and Turbulence in an Urban Canyon. J Appl Meteorol Clim 50, 203-223. 